\title{
Focus point supersymmetry in extended gauge mediation
}

\author{
Ran Ding, ${ }^{a}$ Tianjun Li, ${ }^{b, c}$ Florian Staub ${ }^{d}$ and Bin Zhu ${ }^{a}$ \\ ${ }^{a}$ School of Physics, Nankai University, \\ Tianjin 300071, P.R. China \\ ${ }^{b}$ State Key Laboratory of Theoretical Physics and Kavli Institute for Theoretical Physics (KITPC), \\ Institute of Theoretical Physics, Chinese Academy of Sciences, Beijing 100190, P.R. China \\ ${ }^{c}$ School of Physical Electronics, University of Electronic Science and Technology of China, \\ Chengdu 610054, P.R. China \\ ${ }^{d}$ Bethe Center for Theoretical Physics $\&$ Physikalisches Institut der Universität Bonn, \\ Nußallee 12, 53115 Bonn, Germany \\ E-mail: dingran@mail.nankai.edu.cn, tli@itp.ac.cn, \\ fnstaub@th.physik.uni-bonn.de, zhubin@mail.nankai.edu.cn
}

ABSTRACT: We propose a small extension of the minimal gauge mediation through the combination of extended gauge mediation and conformal sequestering. We show that the focus point supersymmetry can be realized naturally, and the fine tuning is significantly reduced compared to the minimal gauge mediation and extended gauge mediation without focus point. The Higgs boson mass is around $125 \mathrm{GeV}$, the gauginos remain light, and the gluino is likely to be detected at the next run of the LHC. However, the multi-TeV squarks is out of the reach of the LHC. The numerical calculation for fine-tuning shows that this model remains natural.

KeYWORds: Supersymmetry Phenomenology

ArXiv EPRINT: 1312.5407v2 


\section{Contents}

1 Introduction 1

2 Focus points SUSY in Yukawa mediation 3

2.1 Model description 3

2.2 Analytical derivation of focus point SUSY 5

2.3 Reducing gaugino mass fine-tuning via conformal sequestering 6

3 Naturalness and phenomenology 10

4 Conclusion $\quad 16$

$\begin{array}{ll}\text { A Conventions and one-loop RGEs } & 18\end{array}$

$\begin{array}{ll}\text { B General derivation of focus point formula } & 21\end{array}$

\section{Introduction}

The discovery of the Standard Model (SM) like Higgs boson [1, 2] with $m_{h} \simeq 125 \mathrm{GeV}$ has profound implications on naturalness for the minimal supersymmetric standard model (MSSM). In the context of the MSSM there is a strict upper bound on the light Higgs mass at tree level given by $m_{h} \leq m_{Z}$ (see e.g. [3, 4] and references therein). Thus, the large radiative corrections, mainly from (s)tops, are necessary to lift $m_{h}$ to the desired range of about $123-129 \mathrm{GeV}$. The dominant one-loop corrections can be approximated as [5]

$$
m_{h}^{2}=m_{Z}^{2} \cos ^{2} 2 \beta+\frac{3 m_{t}^{4}}{4 \pi^{2} v^{2}}\left(\log \left(\frac{M_{S}^{2}}{m_{t}^{2}}\right)+\frac{X_{t}^{2}}{M_{S}^{2}}\left(1-\frac{X_{t}^{2}}{12 M_{S}^{2}}\right)\right) .
$$

Here, $M_{S}=\sqrt{m_{\tilde{t}_{1}} m_{\tilde{t}_{2}}}$ is the supersymmetry (SUSY) scale defined as the geometric mean of the two stop masses, $m_{t}$ is the running top quark mass, and $X_{t}$ parametrizes the left-right mixing in the stop sector. One widely used possibility to maximize these corrections is to consider a maximal mixing $\left(X_{t} \sim \sqrt{6} M_{S}\right)$ in the stop sector while assuming only moderately large stop masses, see for instance $[6,7]$ and references therein. In that case and including two- and three-loop corrections [8-13] it is possible to explain the Higgs mass with stop mass around $1 \mathrm{TeV}$. However, it has recently been pointed out that a maximal mixing in the stop sector can lead to a global minimum in the scalar potential at which charge and color are broken by vacuum expectations values (VEVs) of the stops [14-16]. The electroweak vacuum will only be metastable and could decay in a cosmological short time. Thus, one is tempted to choose the other possibility to enhance the radiative corrections by using heavier stop masses but keeping the left-right mixing small. In order to accommodate for a 
Higgs mass in the desired range, stop masses $\geq 5 \mathrm{TeV}$ are needed in this scenario. Together with the lack of a signal of any new physics at the LHC this raises uncomfortable issues with naturalness which is widely discussed in the literatures [17-54].

One can easily understand this issue from the tree-level condition of electroweak symmetry breaking (EWSB) which relates the Higgs soft-breaking masses, the $\mu$ parameter and $m_{Z}$. For $\tan \beta \geq 5$ the condition can be expressed by

$$
m_{Z}^{2} \approx-2\left(\mu^{2}+m_{H_{u}}^{2}\left[m_{w}\right]\right) .
$$

Often one does not take $m_{H_{u}}^{2}\left[m_{w}\right]$ but $m_{H_{u}}^{2}[\Lambda]$ as input. $\Lambda$ is the scale where SUSY is broken by some interactions with a hidden sector. The values for $m_{H_{u}}^{2}$ at $m_{w}$ and $\Lambda$ are connected by the renormalization group equations (RGEs). For the evaluation of $m_{H_{u}}^{2}$ the stop masses play an important role because of the size of the top Yukawa coupling $y_{t}$. One finds the relation $m_{H_{u}}^{2}\left[m_{w}\right]=m_{H_{u}}^{2}[\Lambda]+\delta m_{H_{u}}^{2}$ with

$$
\delta m_{H_{u}}^{2} \sim-\frac{3 y_{t}^{2}}{8 \pi^{2}}\left(m_{Q_{3}}^{2}+m_{U_{3}}^{2}+A_{t}^{2}\right) \log \left(\frac{\Lambda^{2}}{m_{w}^{2}}\right) .
$$

In this approximation, we have only considered the third generation Yukawa couplings but neglecting contributions from gaugino masses. The large contributions of the stop masses to the running of $m_{H_{u}}^{2}$ demand some fine-tuning of the fundamental parameters to get viable EWSB. To quantify this fine-tuning different measures have been introduced. We are using throughout this work the one proposed by Barbieri-Giudice [17, 55]

$$
\Delta_{\mathrm{BG}} \equiv \max \left\{\Delta_{a}\right\} \quad \text { where } \quad \Delta_{a} \equiv \frac{\partial \log m_{z}^{2}}{\partial \log a} .
$$

$a$ are the fundamental parameters in the theory. For the constrained MSSM (CMSSM) [56] one takes $a \in\left\{m_{0}^{2}, m_{1 / 2}^{2}, A_{0}^{2}, \mu^{2}, B \mu\right\}$. In this measure the overall fine-tuning of the MSSM in the context of squark masses above $5 \mathrm{TeV}$ and small mixing is expected to be above $10^{4}[57]$.

This large fine-tuning in the MSSM has triggered a lot of interests in models which already increase the tree-level Higgs mass by new contributions from F- or D-terms [58-63]. Especially in singlets extensions like the NMSSM [64-68], GNMSSM [69-73] or DiracNMSSM [74, 75], the fine-tuning is several orders smaller than in the MSSM.

However, also in the MSSM exists parameter regions in which the fine-tuning becomes significantly smaller by one to two orders compared the general expectations. These are the focus point (FP) regions $[27,29,76-78]$. In FP supersymmetry (SUSY), $m_{H_{u}}^{2}\left[m_{w}\right]$ is generated naturally and to a large extent insensitive to the variations of fundamental parameters at the scale $\Lambda$. Besides FP SUSY in the CMSSM, there are also investigations in other SUSY-breaking models including gauge-mediated supersymmetry breaking (GMSB) [79-81], models with large gaugino masses [44, 82-84], and hyperbolic branch SUSY $[85,86]$. We are going to consider here SUSY breaking in the visible sector triggered by gauge interactions.

Already the minimal version of the gauge mediated supersymmetry breaking (GMSB) [87-95] has the appealing features that it softens the flavor problem present in 


\begin{tabular}{|c|c|c|c|}
\hline & $\mathrm{SU}(3)_{C}$ & $\mathrm{SU}(2)_{L}$ & $\mathrm{U}(1)_{Y}$ \\
\hline$\Phi_{1}$ & 1 & 2 & $\frac{1}{2}$ \\
$\tilde{\Phi}_{1}$ & 1 & 2 & $-\frac{1}{2}$ \\
$\Phi_{2}$ & 3 & 1 & $-\frac{1}{3}$ \\
$\tilde{\Phi}_{2}$ & $\overline{3}$ & 1 & $\frac{1}{3}$ \\
$\tilde{\Phi}_{3}$ & 1 & 1 & 0 \\
\hline
\end{tabular}

Table 1. Representations of the messenger fields under the Standard Model gauge group.

gravity mediated SUSY breaking scenarios [96]. On the other hand the minimal GMSB has became unattractive after the Higgs discovery since the $A$-parameters are only generated at the two loop level and usually negligible. Hence, even larger stop masses are needed than in the CMSSM with moderate $A_{0}$ to explain the Higgs mass [97]. This problem can be circumvented to some extent by either extending the gauge groups of the messenger sector $[98,99]$ or by adding superpotential interactions between the matter and messenger fields [100-107].

We are going the second way. In this work we propose a small extension of the minimal GMSB where one Higgs doublet interacts in the superpotential with two messenger fields. In addition, conformal sequestering with negative anomalous dimension is used to suppress the gaugino masses and A-terms. We find that this model has a generic focus point. The simplicity of our model is a main improvement compared to previous attempts to combine GMSB and FP SUSY [79]. So far these models have been very baroque and needed a complicated SUSY breaking mechanism. We will see that in the model presented here the fine-tuning issue is significantly alleviated compared to the minimal GMSB and the model remains natural. Using a precise, numerical setup we find that this model has a fine tuning of about 1000 .

This paper is organized as follows. In section 2, we present the details of our model and derive the analytic solution for FP SUSY. In section 3, we consider the numerical studies of this model. The corresponding fine-tuning measure and phenomenology is discussed in details. We conclude in section 4 . The appendix contains two parts. In A, the conventions and one-loop RGEs are given. In B, we derive the important formula which plays a crucial role in determining FP SUSY.

\section{Focus points SUSY in Yukawa mediation}

\subsection{Model description}

In this paper, we propose an economic and complete model to achieve FP SUSY in GMSB. The messenger sector of our model consists of a pair $(\mathbf{5}, \overline{\mathbf{5}})$ and an singlet under SU(5). Thus, the gauge coupling unification is preserved, and there is no Landau pole below the unification scale because of the small messenger sector. The messenger fields and their quantum numbers with respect to $\mathrm{SU}(3)_{C} \times \mathrm{SU}(2)_{L} \times \mathrm{U}(1)_{Y}$ are summarized in table 1 . In 
the matter sector we have the common superfields of the MSSM. Their superpotential is

$$
W_{\mathrm{MSSM}}=Y_{u} U Q H_{u}+Y_{d} D Q H_{d}+Y_{e} E L H_{d}+\mu H_{u} H_{d} .
$$

In addition, we introduce an interaction between $H_{u}$ and two messenger fields. The superpotential terms involving the messengers are

$$
W_{H \Phi}=X \Phi_{i} \tilde{\Phi}_{i}+\lambda_{u} H_{u} \Phi_{3} \tilde{\Phi}_{1}
$$

Finally, SUSY is broken by some strong interactions in the hidden sector which we leave unspecified. These interactions cause a VEV for $X$ in its scalar and auxiliary components

$$
X \rightarrow M_{m}+\theta^{2} F
$$

and we define $\Lambda \equiv F / M_{m}$. The soft gaugino masses are created by one-loop interactions with the messenger and expected to be $O\left(g_{i}^{2} / 16 \pi^{2} \Lambda\right)$. In absence of any superpotential terms between messenger and MSSM fields, the squared SUSY breaking soft scalar mass terms arise at the two-loop level and are generically of $O\left(\left(g_{i}^{2} / 16 \pi^{2}\right)^{2} \Lambda^{2}\right)$ [108]. In addition to the common contributions from the gauge interactions with the messengers the softterm for $H_{u}$ receives contributions proportional to some power of $\lambda_{u}$ from the interaction given in eq. (2.2). These contributions appear at one- and two-loops. In order to suppress the negative one-loop corrections a large mediation scale of $10^{8} \mathrm{GeV}$ is needed [109]. At two-loop $m_{H_{u}}^{2}$ receives a shift of the form

$$
\Delta m_{H_{u}}^{2} \sim \lambda_{u}^{4}-\lambda_{u}^{2} g^{2}
$$

A precise expression for $\Delta m_{H_{u}}^{2}$ will be derived in section 2.2. The FP SUSY requires $m_{H_{u}}^{2}$ to be comparable with squarks soft terms, i.e., a sizable positive $\Delta m_{H_{u}}^{2}$ is needed. In the messenger sectors larger than the one discussed here, the superpotential interactions between the Higgs fields and messengers charged under $\mathrm{SU}(3)_{C}$ might be allowed. However, those terms would cause negative contribution $\sim \lambda^{2} g_{3}^{2}$. This make the minimal model even more attractive.

Here is a comment on the $A$-terms at place. The extra interaction between Higgs and messenger superfields is often used to generate large A-terms as well. This enhances the Higgs mass and improves the fine-tuning. This setup has been already widely studied in the literatures, see e.g. refs. [105, 109-119]. However, in this paper we assume that the gaugino masses and all $A$-terms are suppressed through conformal sequestering as discussed below. Small gaugino masses are necessary to obtain a SUSY focus point and to reduce the fine-tuning as well. To obtain a focus point the gaugino contributions should be suppressed compared to the sfermion contributions. However, this can't be achieved in the minimal gauge mediation where the gaugino masses are of the same order as sfermion masses. Thus, we consider the conformal sequestering in which gaugino masses are suppressed compared to sfermion masses. In conformal sequestering the gaugino masses are relatively light compared to the other masses because of large negative anomalous dimensions. We explain this in detail in section 2.3 . 
In principle one could keep the $A$-terms large using conformal sequestering while only suppressing the gaugino masses. If $A_{t}$ would not be suppressed, the model will become FP SUSY with large A-term, which greatly improves the fine-tuning since it is easier to obtain $m_{h} \simeq 125 \mathrm{GeV}$. We checked and found FP SUSY also including $A_{t}$. However, this choice is not natural because gaugino masses and $A_{t}$ should be treated at the same status. Therefore, we have not investigated this possibility further. As a result, the maximal mixing scenario could not be achieved and stop will be very heavy in order to satisfy $m_{h}=125 \mathrm{GeV}$. In our setup stop masses of several $\mathrm{TeV}$ are needed and the overall fine-tuning is around 3000, which is well accepted $[120,121]$ and a big improvement compared to the minimal GMSB.

\subsection{Analytical derivation of focus point SUSY}

The soft spectra of the model under considerations can be easily computed via the general formula given in ref. [113]. Applied to our messenger sector and the interaction given in eq. (2.2), the soft-breaking masses for all scalars at messenger scale are given

$$
\begin{aligned}
\tilde{m}_{H_{d}}^{2} & =\tilde{m}_{L}^{2} \\
\tilde{m}_{H_{u}}^{2} & =n_{5} \frac{3}{10}\left(\mathrm{a}_{1}^{2}+5 \mathrm{a}_{2}^{2}\right) \Lambda^{2} f\left(\frac{\Lambda}{M_{m}}\right)+\left(-\frac{3}{5} \mathrm{a}_{1}-3 \mathrm{a}_{2}\right) \Lambda^{2} n_{5} \alpha_{\lambda}+\Lambda^{2}\left(n_{5}^{2}+3 n_{5}\right) \alpha_{\lambda}^{2}, \\
\tilde{m}_{Q}^{2} & =n_{5} \frac{1}{30}\left(\mathrm{a}_{1}^{2}+45 \mathrm{a}_{2}^{2}+80 \mathrm{a}_{3}^{2}\right) \Lambda^{2} f\left(\frac{\Lambda}{M_{m}}\right)-n_{5} Y_{t} \alpha_{\lambda} \Lambda^{2} \\
\tilde{m}_{U}^{2} & =n_{5} \frac{8}{15}\left(\mathrm{a}_{1}^{2}+5 \mathrm{a}_{3}^{2}\right) \Lambda^{2} f\left(\frac{\Lambda}{M_{m}}\right)-2 n_{5} Y_{t} \alpha_{\lambda} \Lambda^{2} \\
\tilde{m}_{D}^{2} & =n_{5} \frac{2}{15}\left(\mathrm{a}_{1}^{2}+20 \mathrm{a}_{3}^{2}\right) \Lambda^{2} f\left(\frac{\Lambda}{M_{m}}\right) \\
\tilde{m}_{L}^{2} & =n_{5} \frac{3}{10}\left(\mathrm{a}_{1}^{2}+5 \mathrm{a}_{2}^{2}\right) \Lambda^{2} f\left(\frac{\Lambda}{M_{m}}\right) \\
\tilde{m}_{E}^{2} & =n_{5} \frac{6}{5} \mathrm{a}_{1}^{2} \Lambda^{2} f\left(\frac{\Lambda}{M_{m}}\right) \\
A_{t} & =-\alpha_{\lambda} \Lambda \\
M_{i} & =g\left(\frac{\Lambda}{M_{m}}\right) \mathrm{a}_{i} \Lambda .
\end{aligned}
$$

Here, we used $\mathrm{a}_{i}=g_{i}^{2} / 16 \pi^{2}(i=1,2,3), \alpha_{\lambda}=\lambda_{u}^{2} / 16 \pi^{2}$ and $n_{5}$ is the messenger index of the 5-plets. $f$ and $g$ are loop-functions which can be found in ref. [108]. $g \sim f \sim 1$ holds in the limit $M_{m} \gg \Lambda$.

As we have mentioned before, we are going to suppress A-term usually generated by Yukawa mediation and also gaugino masses by conformal sequestering. Thus, these contributions can be ignored in our analytical attempts to solve the RGEs. In addition, we neglect all Yukawa couplings except the top quark Yukawa coupling. With the relatively small $\tan \beta<20$, we have following simplified limit,

$$
A_{i}, M_{i}, Y_{b}, Y_{\tau} \rightarrow 0 .
$$

To determine the focus point, $\tilde{m}_{H_{u}}^{2}$ at the weak scale should be written as a function of soft spectra at the conformal scale. Actually, this could be easily obtained when we use 
the one-loop RGEs given in appendix A. In the limit (2.6) the RGEs for the Higgs and stop soft-terms are

$$
\begin{aligned}
\frac{d \tilde{m}_{H_{u}}^{2}}{d t} & =-3 Y_{t}\left(\tilde{m}_{H_{u}}^{2}+\tilde{m}_{Q}^{2}+\tilde{m}_{U}^{2}\right), \\
\frac{d \tilde{m}_{Q}^{2}}{d t} & =-Y_{t}\left(\tilde{m}_{H_{u}}^{2}+\tilde{m}_{Q}^{2}+\tilde{m}_{U}^{2}\right), \\
\frac{d \tilde{m}_{U}^{2}}{d t} & =-2 Y_{t}\left(\tilde{m}_{H_{u}}^{2}+\tilde{m}_{Q}^{2}+\tilde{m}_{U}^{2}\right) .
\end{aligned}
$$

The $\beta$-functions of all other soft-scalar masses vanish in the limit (2.6). Eqs. (2.7)-(2.9) can be solved simultaneously and we find

$$
\tilde{m}_{H_{u}}^{2}[t]=\frac{1}{2}\left(\tilde{m}_{H_{u}}^{2}[0](\mathcal{I}+1)+(\mathcal{I}-1)\left(\tilde{m}_{Q}^{2}[0]+\tilde{m}_{U}^{2}[0]\right)\right)
$$

with

$$
\mathcal{I}=\exp \left(-6 \int_{0}^{t} Y_{t}\left[t^{\prime}\right] d t^{\prime}\right)
$$

where $\mathcal{I}$ is computed in appendix B. The FP SUSY is found at $\tilde{m}_{H_{u}}^{2}[t]=0$, which requires

$$
\frac{\tilde{m}_{H_{u}}^{2}[0]}{\tilde{m}_{Q}^{2}[0]+\tilde{m}_{U}^{2}[0]}=\frac{1-\mathcal{I}}{1+\mathcal{I}} .
$$

It has been proven in ref. [79] that the minimal gauge mediation cannot provide the required ratio. The reason is that $\tilde{m}_{H_{u}}^{2}$ is significantly smaller than squarks soft-term in the minimal gauge mediation because of the dominant contributions from the strongly interacting messengers. However, through the Yukawa mediation, the extra two-loop positive contribution for $\tilde{m}_{H_{u}}^{2}$ and negative contributions to $\tilde{m}_{Q}^{2} / \tilde{m}_{U}^{2}$ are combined to yield a realistic model with focusing behavior. For convenience, we define the required and actually value of eq. (2.12) as

$$
y_{\text {req }}=\frac{1-\mathcal{I}}{1+\mathcal{I}}, \quad y_{\text {act }}=\frac{\tilde{m}_{H_{u}}^{2}[0]}{\tilde{m}_{Q}^{2}[0]+\tilde{m}_{U}^{2}[0]},
$$

while the ratio is given by

$$
\rho=\frac{y_{\text {req }}}{y_{\text {act }}}
$$

It is easy to see from figure 1 that $\log \rho \simeq 0$ can be naturally satisfied for moderate values of $\alpha_{\lambda}$ and a wide range in $M_{*}$.

\subsection{Reducing gaugino mass fine-tuning via conformal sequestering}

In the previous section 2 the suppressed gaugino masses and A-terms have been assumed in order to generate FP SUSY. Here, we present a possible origin of this suppression. To this end we follow previous studies of conformal sequestering in terms of the effective field theory below the messenger scale $M_{m}$ [122-127]. In this setup, the visible and hidden sectors are coupled through irrelevant operators in the Kähler potential. We summarize here the main idea and refer the interested reader for many more details to ref. [124]. 


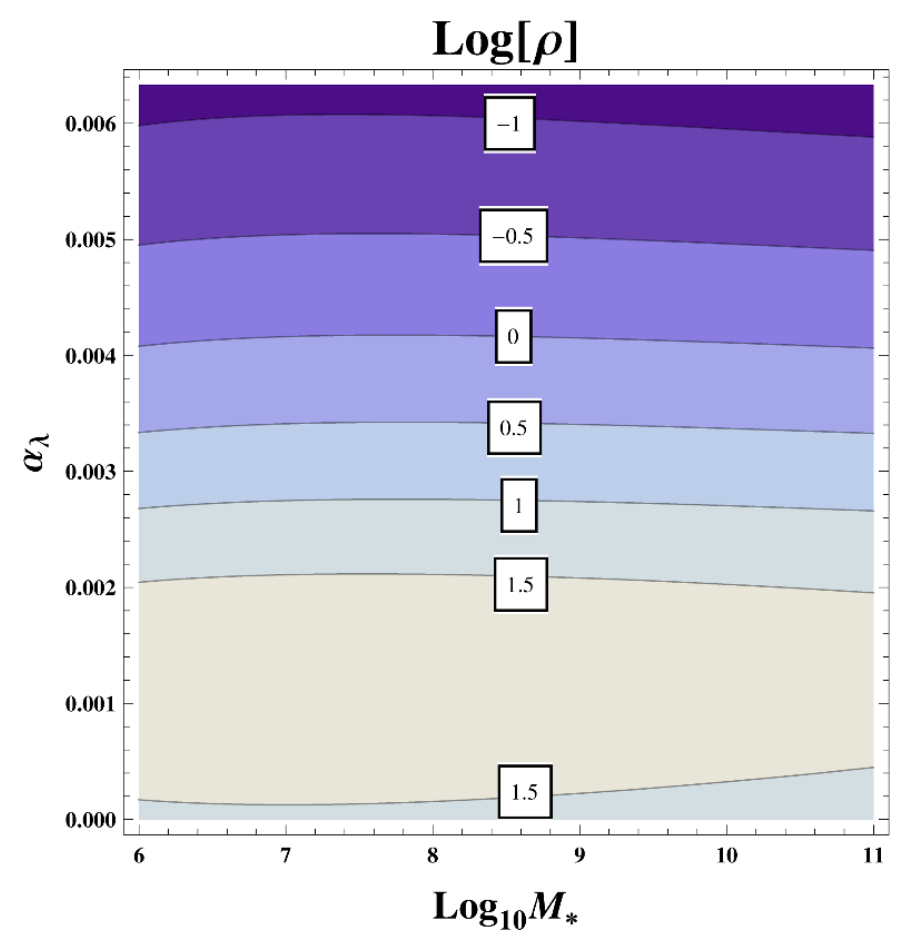

Figure 1. In this figure, we take $\alpha_{e m}^{-1}=127.931993, \alpha_{s}=0.1720, m_{Z}=91.1876$, top quark pole mass $m_{t}=172.9$, and $\tan \beta=10$. Meanwhile, the high scale input includes $n_{5}=1$. In addition, we point out that for arbitrary $n_{5}<5$, the focus point SUSY is generic.

In gauge mediation the gaugino and scalar masses are generated after integrating out the messenger multiplets at respectively one- and two-loop level. The effective interactions for the gauge and matter multiplets in the MSSM with a singlet in the hidden sector $X$ are

$$
\mathcal{L}_{\text {eff }}=\left[\int d^{2} \theta \sum_{a=1}^{3} \frac{1}{2} c_{\lambda}^{a} \frac{X}{M_{m}} \mathcal{W}^{a \alpha} \mathcal{W}_{\alpha}^{a}+h . c .\right]-\int d^{4} \theta \sum_{\tilde{f}} c_{m^{2}}^{\tilde{f}} \frac{X^{\dagger} X}{M_{m}^{2}} \tilde{f}^{\dagger} \tilde{f} .
$$

Here, $\mathcal{W}^{a \alpha}$ (with $a=1,2,3$ ) are the field strength superfields for the SM gauge sector and $\tilde{f} \in\left\{q, u, d, l, e, H_{u}, H_{d}\right\}$. The coefficients $c_{\lambda}^{a}$ appear at one loop and $c_{m^{2}}^{\tilde{f}}$ at two loop. The precise definitions of these coefficients are given in ref. [128].

When the hidden sector enters the conformal regime at some scale $M_{*},{ }^{1}$ the two terms in eq. (2.15) receive large corrections from wave function renormalization. The effective Lagrangian is then given at some renormalization scale $\mu_{R}\left(\right.$ with $\left.\mu_{R}<M_{m}\right)$ by

$$
\mathcal{L}_{\text {eff }}=\left[\int d^{2} \theta \sum_{a=1}^{3} \frac{1}{2} c_{g} Z_{X}^{-1 / 2} \frac{X}{M_{m}} \mathcal{W}^{a \alpha} \mathcal{W}_{\alpha}^{a}+\text { h.c. }\right]-\int d^{4} \theta \sum_{\tilde{f}} c_{m^{2}}^{\tilde{f}} Z_{X}^{-1} Z_{|X|^{2}} \frac{X^{\dagger} X}{M_{m}^{2}} \tilde{f}^{\dagger} \tilde{f} .
$$

From this equation it can be seen that the wave function renormalization constants $Z_{X}^{-1 / 2}$ and $Z_{|X|^{2}}$ can be used to suppress either the scalar or the gaugino soft masses. At the

\footnotetext{
${ }^{1}$ Noting we use $M_{*}$ denotes the conformal scale to avoid confusion with the parameter $\Lambda$ in GMSB, which corresponds to $\Lambda_{*}$ in ref. [124].
} 
conformal scale $M_{*}$ the renormalization constants are given by

$$
Z_{X}[0]=\left(\frac{M_{m}}{M_{*}}\right)^{3 R(X)-2}, \quad Z_{|X|^{2}}[0]=\left(\frac{M_{*}}{M_{m}}\right)^{\gamma} .
$$

Here, $[0]$ indicates the quantities which are evaluated at the conformal scale. In the Higgs sector, the $\mu$ term, $B_{\mu}$ and $A_{H_{d}}$ cannot be generated at the messenger scale because the messenger couple only to $H_{u}$. Thus, the effective Lagrangian at conformal scale is given by

$$
\mathcal{L}_{\mathrm{eff}}=-\left[\int d^{4} \theta c_{A_{u}} Z_{X}^{-1 / 2} \frac{X}{M_{m}} H_{u} H_{u}^{\dagger}\right]-\int d^{4} \theta Z_{X}^{-1} Z_{|X|^{2}} \frac{X^{\dagger} X}{M_{m}^{2}}\left(H_{u} H_{u}^{\dagger}+H_{d} H_{d}^{\dagger}\right) .
$$

Using eqs. (2.16) to (2.18), the soft SUSY spectra at the conformal scale $M_{*}$ can be related to those at messenger scale $M_{m}$ via

$$
M_{i}[0]=Z_{X}^{-1 / 2} M_{i}\left[t_{m}\right], \quad A_{t}[0]=Z_{X}^{-1 / 2} A_{t}\left[t_{m}\right], \quad m_{f}^{2}[0]=Z_{X}^{-1} Z_{|X|^{2}} m_{\tilde{f}}^{2}\left[t_{m}\right],
$$

with $t_{m}=2 \log \left(\frac{M_{*}}{M_{m}}\right)$. For $\gamma>0$ and $R(X)>3 / 2$ the conventional conformal sequestering is achieved and one finds $m_{\tilde{f}}^{2} \approx 0$. However, it has been shown in ref. [129] that this type of conformal sequestering is severely constrained by stringent bounds on $\gamma$ from internal consistency of the hidden sector superconformal field theory $(\mathrm{SCFT})$. For $\operatorname{dim}(X) \simeq 1$ self-consistent condition requires $\gamma=\operatorname{dim}\left(X X^{\dagger}\right)-2 \operatorname{dim}(X)<0$. This forbids the positive anomalous dimensions and as consequence the sfermion and Higgs masses are enhanced compared to the gaugino masses.

We make use of this suppression of the gaugino masses and A-terms relative to the sfermion and Higgs masses in the case of $\gamma<0$. For this purpose we parametrize

$$
Z_{X}^{-1 / 2}=\eta, \quad Z_{|X|^{2}}=\frac{1}{\epsilon^{2}}, \quad \Lambda_{a}=\frac{\eta}{\epsilon} \Lambda .
$$

Using these definitions we can relate $\Lambda$ and $M_{m}$ to $\Lambda_{a}$ and $M_{*}$ via

$$
\frac{\Lambda}{M_{m}}=\left(\frac{\epsilon \Lambda_{a}}{\eta M_{*}}\right)^{2} \text {. }
$$

We finally end up with the following boundary conditions for the soft masses in our GMSB version of the MSSM

$$
\begin{aligned}
\tilde{m}_{H_{d}}^{2}[0]= & \tilde{m}_{L}^{2}[0] \\
\tilde{m}_{H_{u}}^{2}[0]= & n_{5} \frac{3}{10}\left(\mathrm{a}_{1}[0]^{2}+5 \mathrm{a}_{2}[0]^{2}\right) \Lambda_{a}^{2} f\left(\frac{\eta^{2} \Lambda^{2}}{\epsilon^{2} M_{*}^{2}}\right)+\left(-\frac{3}{5} \mathrm{a}_{1}[0]-3 \mathrm{a}_{2}[0]\right) \Lambda_{a}^{2} n_{5} \alpha_{\lambda} \\
& +\Lambda_{a}^{2}\left(n_{5}^{2}+3 n_{5}\right) \alpha_{\lambda}^{2}, \\
\tilde{m}_{Q}^{2}[0]= & n_{5} \frac{1}{30}\left(\mathrm{a}_{1}[0]^{2}+45 \mathrm{a}_{2}[0]^{2}+80 \mathrm{a}_{3}[0]^{2}\right) \Lambda_{a}^{2} f\left(\frac{\eta^{2} \Lambda_{a}^{2}}{\epsilon^{2} M_{*}^{2}}\right)-n_{5} Y_{t}[0] \alpha_{\lambda} \Lambda_{a}^{2}, \\
\tilde{m}_{U}^{2}[0]= & n_{5} \frac{8}{15}\left(\mathrm{a}_{1}[0]^{2}+5 \mathrm{a}_{3}[0]^{2}\right) \Lambda_{a}^{2} f\left(\frac{\eta^{2} \Lambda_{a}^{2}}{\epsilon^{2} M_{*}^{2}}\right)-2 n_{5} Y_{t}[0] \alpha_{\lambda} \Lambda_{a}^{2}, \\
\tilde{m}_{D}^{2}[0]= & n_{5} \frac{2}{15}\left(\mathrm{a}_{1}[0]^{2}+20 \mathrm{a}_{3}[0]^{2}\right) \Lambda_{a}^{2} f\left(\frac{\eta^{2} \Lambda_{a}^{2}}{\epsilon^{2} M_{*}^{2}}\right),
\end{aligned}
$$




$$
\begin{aligned}
\tilde{m}_{L}^{2}[0] & =n_{5} \frac{3}{10}\left(\mathrm{a}_{1}[0]^{2}+5 \mathrm{a}_{2}[0]^{2}\right) \Lambda_{a}^{2} f\left(\frac{\eta^{2} \Lambda_{a}^{2}}{\epsilon^{2} M_{*}^{2}}\right), \\
\tilde{m}_{E}^{2}[0] & =n_{5} \frac{6}{5} \mathrm{a}_{1}[0] 2 \Lambda_{a}^{2} f\left(\frac{\eta^{2} \Lambda_{a}^{2}}{\epsilon^{2} M_{*}^{2}}\right), \\
A_{t}[0] & =\epsilon \alpha_{\lambda}[0] \Lambda_{a}, \\
M_{i}[0] & =\epsilon \mathrm{a}_{i}[0] \Lambda_{a} g\left(\frac{\eta^{2} \Lambda_{a}^{2}}{\epsilon^{2} M_{*}^{2}}\right) .
\end{aligned}
$$

To sum up, the free parameters of this model are $\left\{\eta, \epsilon, n_{5}, \Lambda_{a}, \lambda_{u}, M_{*}, \tan \beta, \operatorname{sign}(\mu)\right\}$. Since $\eta$ only enters in the loop function $f(x)$ and $g(x)$ one can impose $\eta=\epsilon$ for simplicity to remove one degree of freedom, one found that in this case the gaugino masses are always suppressed relative to the scalar masses. In total, there are six free parameters and one sign in this model

$$
\left\{\epsilon, n_{5}, \Lambda_{a}, \lambda_{u}, M_{*}, \tan \beta, \operatorname{sign}(\mu)\right\}
$$

Without leading to confusion, the parameter $\Lambda_{a}$ could is identified with $\Lambda$ after imposing $\eta=\epsilon$, and

$$
M_{m} \Lambda=M_{*}^{2}
$$

Above equation is valid for any scale of $M_{m}$ and $\Lambda$. It is interesting to discuss the ranges of $M_{m}, \Lambda$ and $M_{*}$. First note that $m_{h}$ is most relative to $\Lambda$ scale, $125 \mathrm{GeV}$ Higgs requires $\Lambda$ fall into the range $10^{4}<\Lambda<10^{6} \mathrm{GeV}$. Meanwhile, to safely ignore the gravity mediation effect, one natural choice for upper bound of messenger scale is $M_{m}<10^{16} \mathrm{GeV}$. one thus obtain the range of $M_{*}$ from eq. (2.24) as $10^{6}<M_{*}<10^{11} \mathrm{GeV}$. These parameter range for $\Lambda$ and $M_{*}$ are applied to our numerical calculation in next section.

For now, we only consider the scenario of $\eta=\epsilon$. We could also consider the effect of $\eta$ when it is not removed. Through the parameter transformation (2.20). We found $\eta$ only appears in the loop function $f_{\mathrm{GMSB}}$ and $g_{\mathrm{GMSB}}$, where

$$
\begin{aligned}
f_{\mathrm{GMSB}}= & \frac{(1-x)\left(-2 \operatorname{Li}_{2}\left(\frac{x}{x-1}\right)+\frac{1}{2} \operatorname{Li}_{2}\left(\frac{2 x}{x-1}\right)+\log (1-x)\right)}{x^{2}} \\
& +\frac{(x+1)\left(-2 \operatorname{Li}_{2}\left(\frac{x}{x+1}\right)+\frac{1}{2} \operatorname{Li}_{2}\left(\frac{2 x}{x+1}\right)+\log (x+1)\right)}{x^{2}} \\
g_{\mathrm{GMSB}}= & \frac{(1-x) \log (1-x)}{x^{2}}+\frac{(x+1) \log (x+1)}{x^{2}}
\end{aligned}
$$

and $x$ is now defined to be $\left(\frac{\epsilon \Lambda_{a}}{\eta M_{*}}\right)^{2}$ rather than $\frac{\Lambda}{M_{m}}$. The dependence on $x$ for $f_{\text {GMSB }}$ and $g_{\text {GMSB }}$ are plotted in figure 2 .

We found that only the ratio $\epsilon / \eta$ is relevant for given $\Lambda_{a}$ and $M_{*}$. Since they appear simultaneously. There are three possibilities to study:

1. $\epsilon=\eta$. That is the main concern of the paper. 


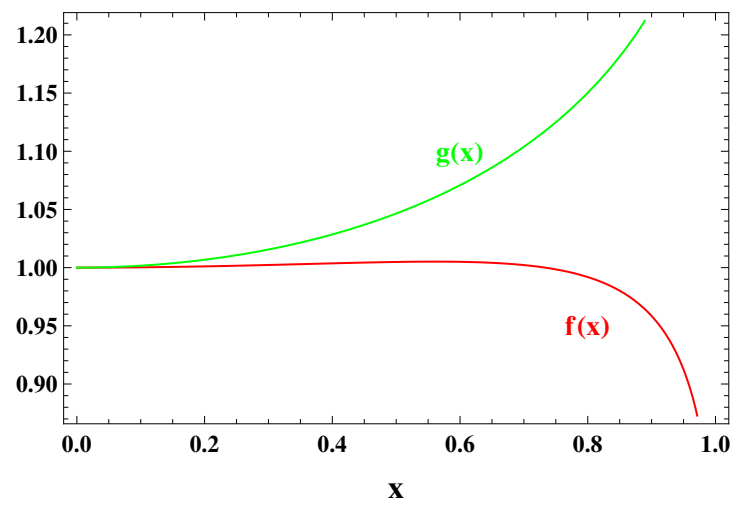

Figure 2. f and g respectively.

2. $\epsilon>\eta$. Given certain $\Lambda_{a}$ and $M_{*}$, the gaugino becomes heavier than that of $\epsilon=\eta$ and sfermion becomes lighter. These effects are inverse to the scenario of conformal sequestering that we adapt. Then the analysis of focus point might be invalid. Here we ignore this choice of parameters.

3. $\epsilon<\eta$. In addition to $\Lambda_{a}<M_{*}$, this choice make $f(x)$ and $g(x)$ degenerate to identity. Thus it has no difference from that of $\epsilon=\eta$.

That is why we take $\epsilon=\eta$ to study. This particular choice of parameter is in fact universal and physical for the soft term determination.

\section{$3 \quad$ Naturalness and phenomenology}

We present in this section our numerical results for the fine tuning in this model and comment on some phenomenological features. For this purpose, we have implemented this model in the Mathematica package SARAH [130-134]. SARAH has been used to create a SPheno $[135,136]$ version for the MSSM with the new boundary conditions for the softterms at the messenger scale. The SARAH generated SPheno version calculates the mass spectrum with the same precision as SPheno 3.2.4 but includes also routines to calculate the fine tuning according to eq. (1.4). In our case the free parameters which influence the fine tuning are slightly different compared to ref. [113] since we have suppressed all one-loop contributions to the soft-masses at the messenger scale to have a sufficiently large $m_{H_{u}}^{2}$. Thus, we calculate the fine tuning with respect to

$$
a \in\left\{\Lambda, \lambda_{u}, \mu, g_{3}, y_{t}\right\}
$$

The null results from SUSY searches at the LHC put severe limits on the allowed masses of the gluino and of the squarks of the first two generations [137, 138]. These limits can roughly be summarized to: (i) $m_{\tilde{g}} \gtrsim 1.5 \mathrm{TeV}$ (for $m_{\tilde{g}} \simeq m_{\tilde{q}}$ ), (ii) $m_{\tilde{g}} \gtrsim 1 \mathrm{TeV}$ (for $\left.m_{\tilde{g}} \ll m_{\tilde{q}}\right)$. In our case the gluino is always much lighter than the squarks because of the suppression from conformal sequestering. Thus, we impose the constraint $m_{\tilde{g}} \gtrsim 1 \mathrm{TeV}$ in addition to $123<m_{h}<129 \mathrm{GeV}$. 
To check the overall fine tuning in our model we have performed a random scan of $2 \times 10^{5}$ points in the following parameter ranges

$$
\begin{aligned}
10^{4} \mathrm{GeV} & <\Lambda_{a}<10^{6} \mathrm{GeV}, \\
10^{6} \mathrm{GeV} & <M_{*}<10^{11} \mathrm{GeV}, \\
0 & <\lambda_{u}<1 \\
0.1 & <\epsilon<1 .
\end{aligned}
$$

The other parameters have been fixed to $\tan \beta=10, n_{5}=1, n_{10}=0$ and $\operatorname{sign}(\mu)=1$. The parameter scans have been performed with SSP [139]. The point with the best fine tuning satisfying the constraints on the SUSY and Higgs masses has a fine tuning of $\Delta=3117$, see for more details table 2. Thus, even if we keep the mixing in the stop very small and need very heavy stops to obtain the correct Higgs, a fine tuning of about 3000 is still very good compared the CMSSM expectations with $A_{0}=0$. Because the electroweak gauge symmetry will not be broken at the weak scale if supersymmetry is not broken, the relevent parameters for fine-tuning measures are $\mu$ and $\Lambda_{a}$. Also, the Yukawa couplings can be calculated in the concrete model building, for example, string models. Thus, we can just consider $\mu$ and $\Lambda_{a}$ for fine-tuning study, and the corresponding fine tuning is reduced to $\Delta \sim 1000$. A benchmark point is given in table 3 .

In figure 5 , we show the fine tuning in the $\left(\lambda_{u}, \Lambda_{a}\right)$ plane for two different combinations of $\epsilon$ and $M_{*}:\left(\epsilon=0.4, M_{*}=8.5 \times 10^{8} \mathrm{GeV}\right)$ and $\left(\epsilon=0.2, M_{*}=5 \times 10^{10} \mathrm{GeV}\right)$. The behavior of the fine tuning can be summarized as follows

1. In the $\left(\lambda_{u}, \Lambda_{a}\right)$ space: for a given value of $M_{*}$ and $\epsilon$, increasing $\Lambda_{a}$ and $\lambda_{u}$ increases the overall fine tuning. The reason is that large $\Lambda_{a}$ and $\lambda_{u}$ increase $\delta m_{H_{u}}^{2}$, see eqs. (1.3) and (2.4).

2. Small values of $\Lambda_{a}$ and $\lambda_{u}: \Delta$ is usually dominated by $\mu$. Since in these regions the RGE effects are most important, the contribution to the fine tuning of $\lambda_{u}$, which only affects the boundary conditions, is negligible. The important parameters are $\Lambda_{a}$ which sets the range of the RGE running and, even more important, the absolute value of $\mu$.

3. moderate $\Lambda_{a}$ and $\lambda_{u}$ : the contribution from $\mu$ and $\Lambda_{a}$ are almost comparable.

4. large $\lambda_{u}$ : if $\lambda_{u}$ becomes large it is always the biggest contributor to fine tuning measure independent of the value of $\Lambda_{a}$. This seems to contradict the requirement of FP SUSY, for which special $\lambda_{u}$ gives rise to the focusing behavior thus reducing the fine tuning. However, small changes in $\lambda_{u}$ lead to sizable changes in the Higgs soft parameter at the threshold scale. The problem would improve if $\lambda_{u}$ has a fixed point for a given $M_{*}$. In that case the fine tuning induced by $\lambda_{u}$ would be completely negligible. We leave this topic for future investigation and accept here the fine tuning with respect to $\lambda_{u}$. Once we eliminate the couplings $y_{t}, g_{3}$ and $\lambda_{u}$ from the fundamental parameters, the corresponding fine-tuning will become 1000, which falls into the regime of natural SUSY. 


\begin{tabular}{|c|c|c|c|c|c|c|}
\hline$\Lambda_{a}(\mathrm{GeV}), M_{*}(\mathrm{GeV}), \lambda_{u}, \epsilon$ & $\Delta_{F T}$ & $A_{t} / M_{S}$ & $m_{\tilde{g}}(\mathrm{GeV})$ & $m_{\tilde{t}_{1}}(\mathrm{GeV})$ & $m_{\tilde{\tau}_{1}}(\mathrm{GeV})$ & $m_{\tilde{\chi}_{1}^{0}}(\mathrm{GeV})$ \\
\hline $8 \times 10^{5}, 8.5 \times 10^{8}, 0.57,0.39$ & 3117 & -0.28 & 2333 & 4299 & 1573 & 436 \\
\hline
\end{tabular}

Table 2. The point with minimal fine tuning in our random scans. The parameter ranges have been chosen according to eq. (3.2).

\begin{tabular}{|c|c|c|c|c|c|c|}
\hline$\Lambda_{a}(\mathrm{GeV}), M_{*}(\mathrm{GeV}), \lambda_{u}, \epsilon$ & $\Delta_{F T}$ & $A_{t} / M_{S}$ & $m_{\tilde{g}}(\mathrm{GeV})$ & $m_{\tilde{t}_{1}}(\mathrm{GeV})$ & $m_{\tilde{\tau}_{1}}(\mathrm{GeV})$ & $m_{\tilde{\chi}_{1}^{0}}(\mathrm{GeV})$ \\
\hline $8.7 \times 10^{5}, 9.5 \times 10^{9}, 0.71,0.27$ & 1127 & -0.28 & 1831 & 3496 & 1692 & 327 \\
\hline
\end{tabular}

Table 3. Similar to table 2 , but here we only consider the $\mu$ and $\Lambda_{a}$ for tuning measure.

5. For a given value of $M_{*}$ and $\epsilon,\left\{\Lambda, \mu, y_{t}, g_{3}\right\}$ both have similar behavior, i.e., more sensitive to the variation of $\Lambda$ then $\lambda_{u}$, increasing $\Lambda$ increases the overall fine tuning. While the fine tuning for $\lambda_{u}$, on the opposite, more sensitive to itself. The combination of these two different behavior generally has a balance point in the parameter space which corresponds the minimum of the overall fine tuning. That is the most important reason for the overall fine tuning has different minimum for two parameter benchmarks, which could be seen in figure 3 and figure 4 . That explains the different behaviors in different benchmark points of the model. While, in figure 1 , the focus point supersymmetry is proven to be universal, which seems to contradict with the behaviors in figure 3. Indeed, It is not true. In figure 1 , the focus point is determined in the one-loop and semi-analytic analysis of renormalization group equations without considering threshold effects. That means the tiny difference might come from the fact that, SPheno considered threshold effects and solved the two-loop renormalization group equations numerically. In addition to the numerical precision, we think, the biggest difference is that focus point supersymmetry only considered the fine-tuning induced by $\mu$ term which was clearly different from the equation (3.1). Then, the combination effects from equation (3.1) makes the finetuning slightly different

We shall finish some comments on the phenomenological aspects of the focus point in the presented model. For this purpose, we show in the figures 6-8 the contours of relevant SUSY masses in the $\left(\lambda_{u}, \Lambda_{a}\right)$ plane for the same combinations of $\epsilon$ and $M_{*}$ as in figure 5 . As it can be seen in figure 7, the gluino for $\epsilon=0.2$ is well below $2 \mathrm{TeV}$ and then within the reach of the next run of the LHC experiments. The stop is always in the multi TeV range and therefore out of reach. Hence, this model leads naturally to a split SUSY behavior which is widely discussed in literatures, see e.g. [140, 140-144]. In addition, we show the ratio $A_{t} / M_{S}$ which is always small in the entire range.

In figure 8, we consider which parameters are interesting with respect to the dark matter properties of this model: the mass of the lightest neutralinos $m_{\tilde{\chi}_{1}^{0}}$, the mass ratio between stau and neutralino $m_{\tilde{\tau}_{1}} / m_{\tilde{\chi}_{1}^{0}}$ and the mass ratio of the Gravitino and neutralino $\log _{10}\left(m_{\tilde{G}} / m_{\tilde{\chi}_{1}^{0}}\right)$. Here, we take again the same parameter space as in figure 5 . It is 

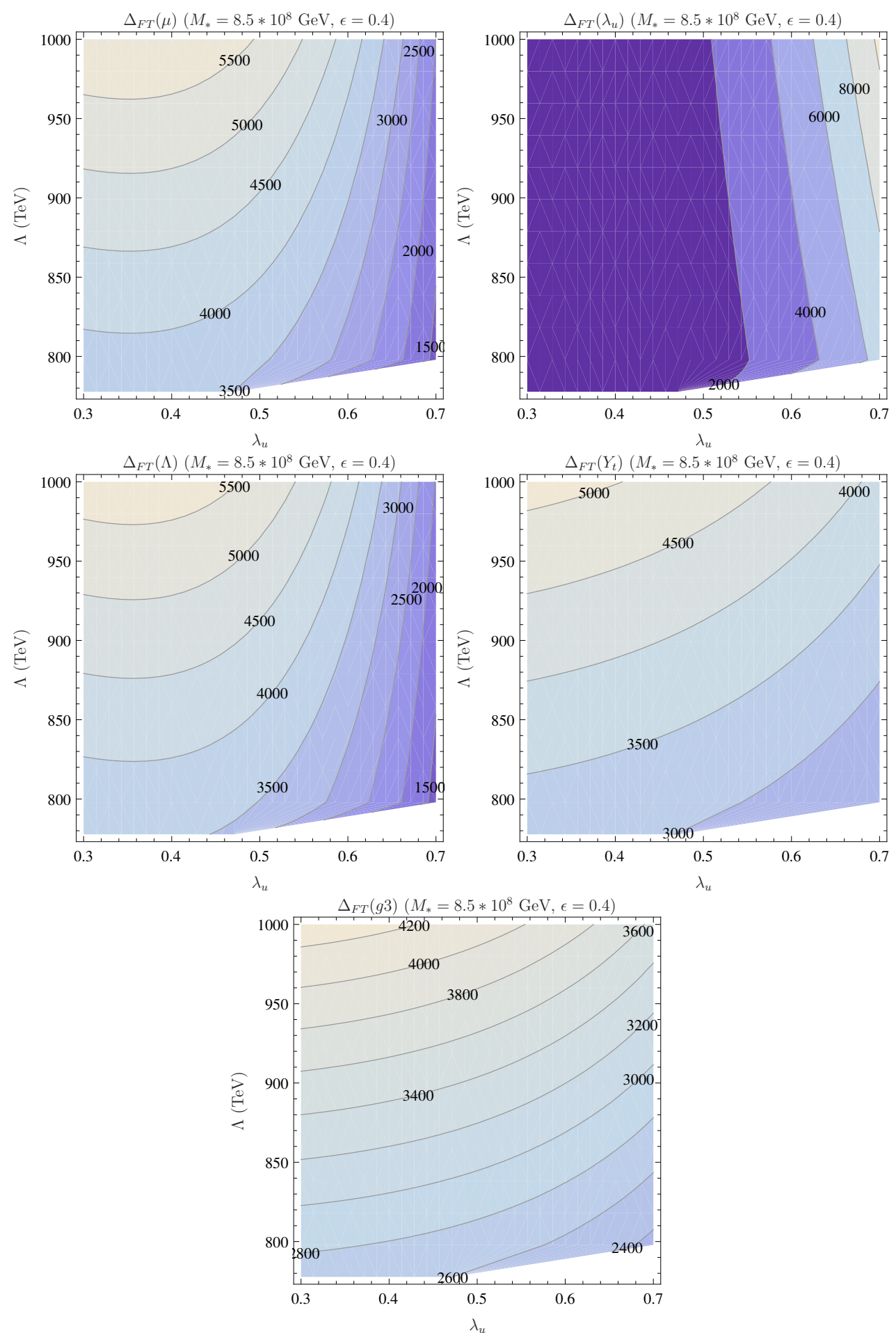

Figure 3. Fine tuning for each parameter in the $\left(\lambda_{u}, \Lambda_{a}\right)$ plane for $\epsilon=0.4, M_{*}=8.5 \times 10^{8} \mathrm{GeV}$. Figure from left to right and from top to bottom are $\Delta_{F T}(\mu), \Delta_{F T}\left(\lambda_{u}\right), \Delta_{F T}(\Lambda), \Delta_{F T}\left(y_{t}\right)$ and $\Delta_{F T}\left(g_{3}\right)$, respectively. The other parameters are fixed to $\tan \beta=10, n_{5}=1, n_{10}=0$ and $\operatorname{sign}(\mu)=1$. 

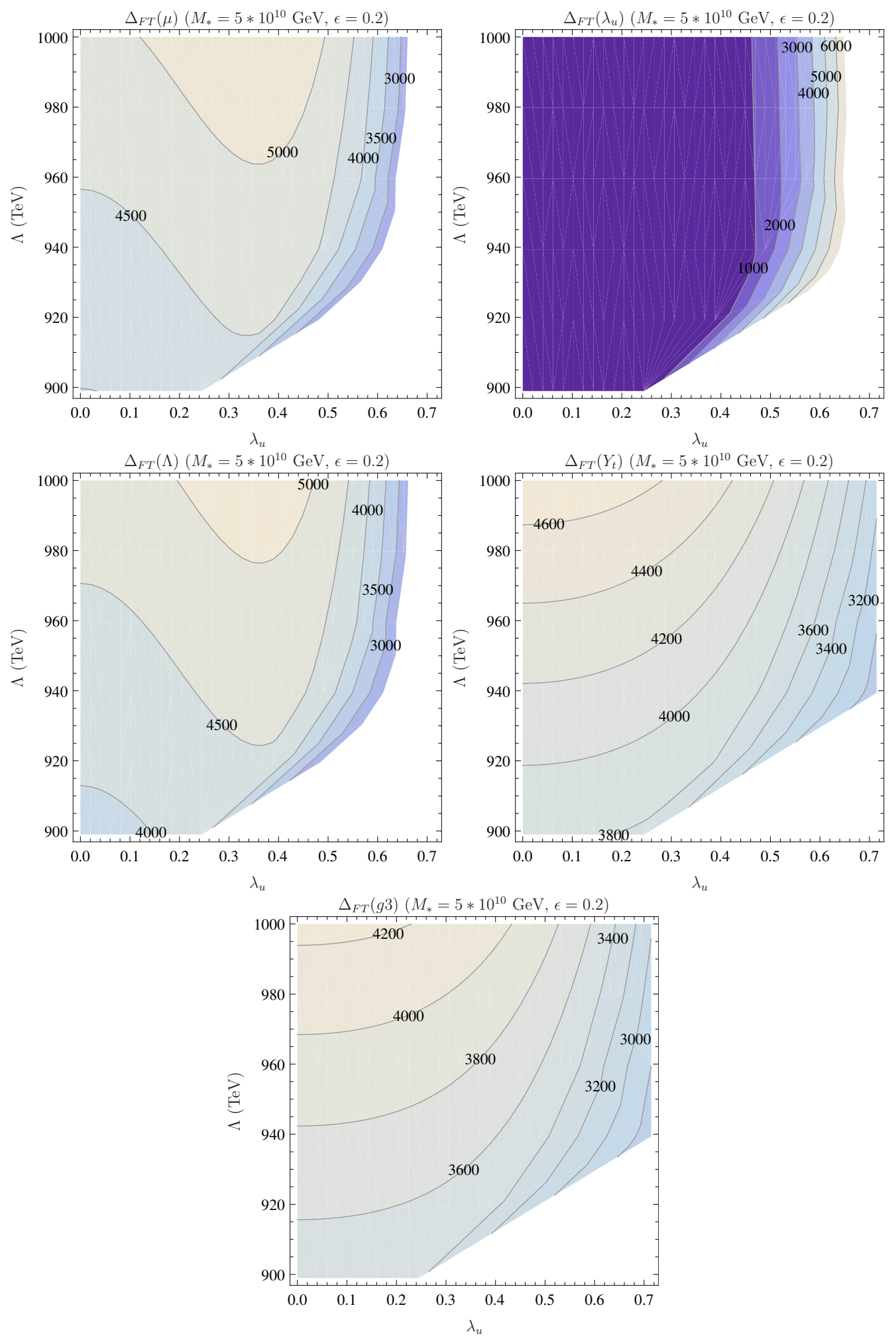

Figure 4. Similar to figure 3, but for $\epsilon=0.2, M_{*}=5 \times 10^{1} 0 \mathrm{GeV}$. 

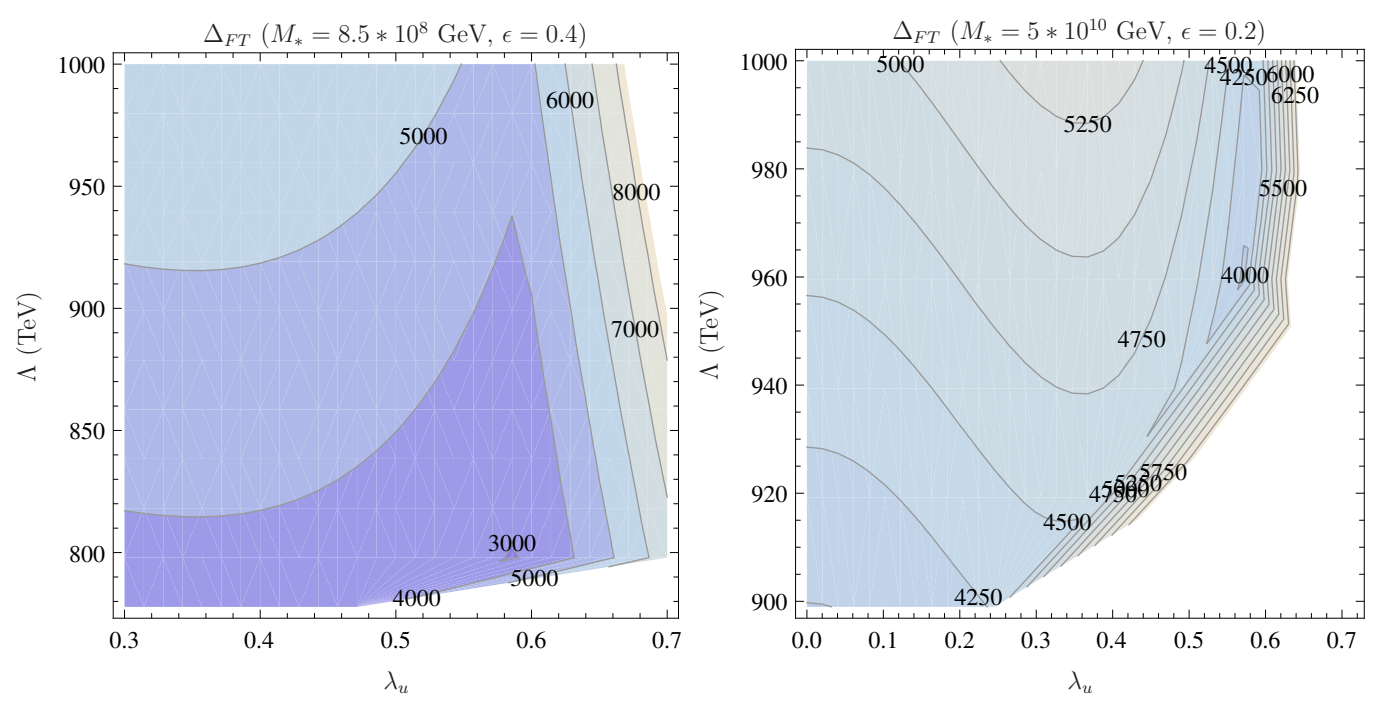

Figure 5. Fine tuning in the $\left(\lambda_{u}, \Lambda_{a}\right)$ plane for $\epsilon=0.4, M_{*}=8.5 \times 10^{8} \mathrm{GeV}$ (left-panel) and $\epsilon=0.2, M_{*}=5 \times 10^{10} \mathrm{GeV}$. The other parameters are fixed to $\tan \beta=10, n_{5}=1, n_{10}=0$ and $\operatorname{sign}(\mu)=1$.

well known that the gravitino is usually the lightest supersymmetric particle (LSP) in conventional GMSB model with a mass of

$$
m_{3 / 2}=\frac{F}{\sqrt{3} M_{\mathrm{Pl}}}
$$

with $F=M_{*}^{2}$. Here, $M_{\mathrm{Pl}}=2.4 \times 10^{18} \mathrm{GeV}$ is the reduced Planck mass. One of the most intriguing feature is that at high $M_{*}$ scale, the LSP in mass spectrum will naturally become the neutralino which is the promising dark matter candidate which does not suffer from the cosmological gravitino problem [145-151]. To demonstrate this feature, we show the mass ratio of the gravitino and neutralino. It can be seen clearly that the neutralino is the LSP in the entire parameter region for $M_{*} \sim 5 \times 10^{10} \mathrm{GeV}$. The mass of the neutralino is of the order of a few hundred $\mathrm{GeV}$, i.e., of the typical range of WIMP (weakly interacting massive particle) candidate for dark matter.

We have only touched here the interesting phenomenological aspects of the model but concentrated on the fine tuning properties. A detailed discussion of the mass spectrum and the dark matter properties of a neutralino LSP will be given elsewhere.

The phenomenology of this model is different from conventional GMSB, since the NLSP (LSP)is neutralino rather than stau in most of the parameter space, which could be seen in figure 9 . In the colored sector, the stops are several TeV to satisfy $m_{h}=125 \mathrm{GeV}$. Therefore all the squarks and sleptons escape the current limits of LHC. In figure 9, the spectrum of the model at best point is given, which implies $H^{0}, A^{0}$ and $H^{ \pm}$are quite heavy so that the Higgs sector is within the decoupling limit and the lightest Higgs properties are those of the Standard Model. In figure 10, the spectra at high conformal scale are given. Here neutralino becomes the LSP, which plays a crucial role in DM research. 

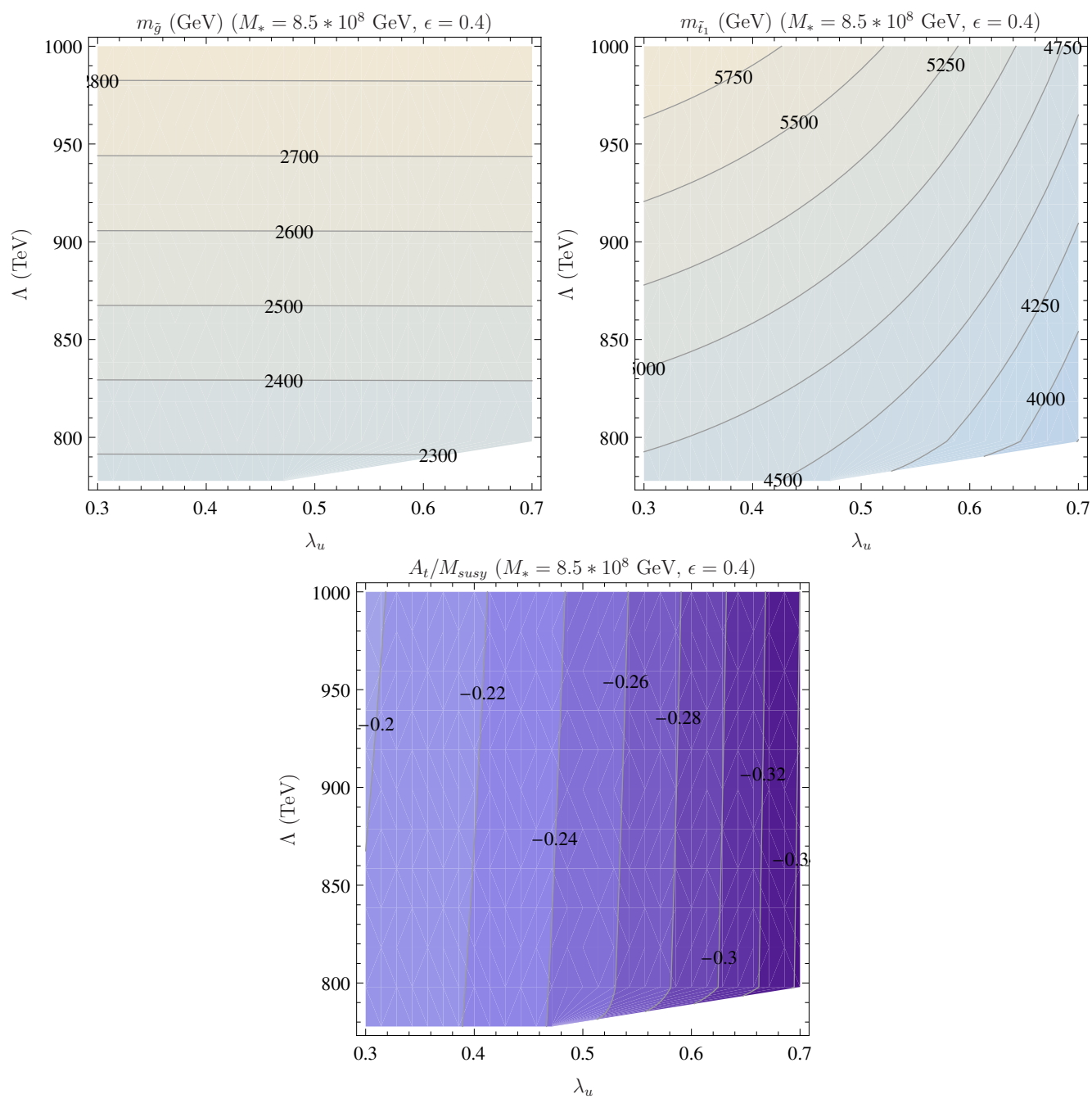

Figure 6. Gluino mass $m_{\tilde{g}}$ (top-left), stop mass $m_{\tilde{t}_{1}}$ (top-right), and stop mixing $A_{t} / M_{S}$ (bootom) in the $\left(\lambda_{u}, \Lambda_{a}\right)$ plane for $M_{*}=8.5 \times 10^{8} \mathrm{GeV}$, and $\epsilon=0.4$. The other parameters are fixed to $\tan \beta=10, n_{5}=1, n_{10}=0$ and $\operatorname{sign}(\mu)=1$.

\section{Conclusion}

The discovery of the Higgs boson with a mass of $m_{h} \simeq 125 \mathrm{GeV}$ raises a challenge for naturalness in the MSSM. In order to alleviate the fine tuning induced by several $\mathrm{TeV}$ stops, we have introduced a model for focus point SUSY in the context of gauge mediation. In contrast to previous attempts to combine gauge mediation and focus point SUSY our model is very simple but keeps the fine tuning under control.

Through the combination of Yukawa mediation and conformal sequestering, we found a calculable model of gauge mediation, which automatically satisfies the minimal flavor violation (MFV). In addition, the $A$-terms in this model are small for the price of heavy stops. However, this also evades possible issues with color and charge breaking minima. Although the suppression of the $A$-terms, the corresponding fine tuning in this model 

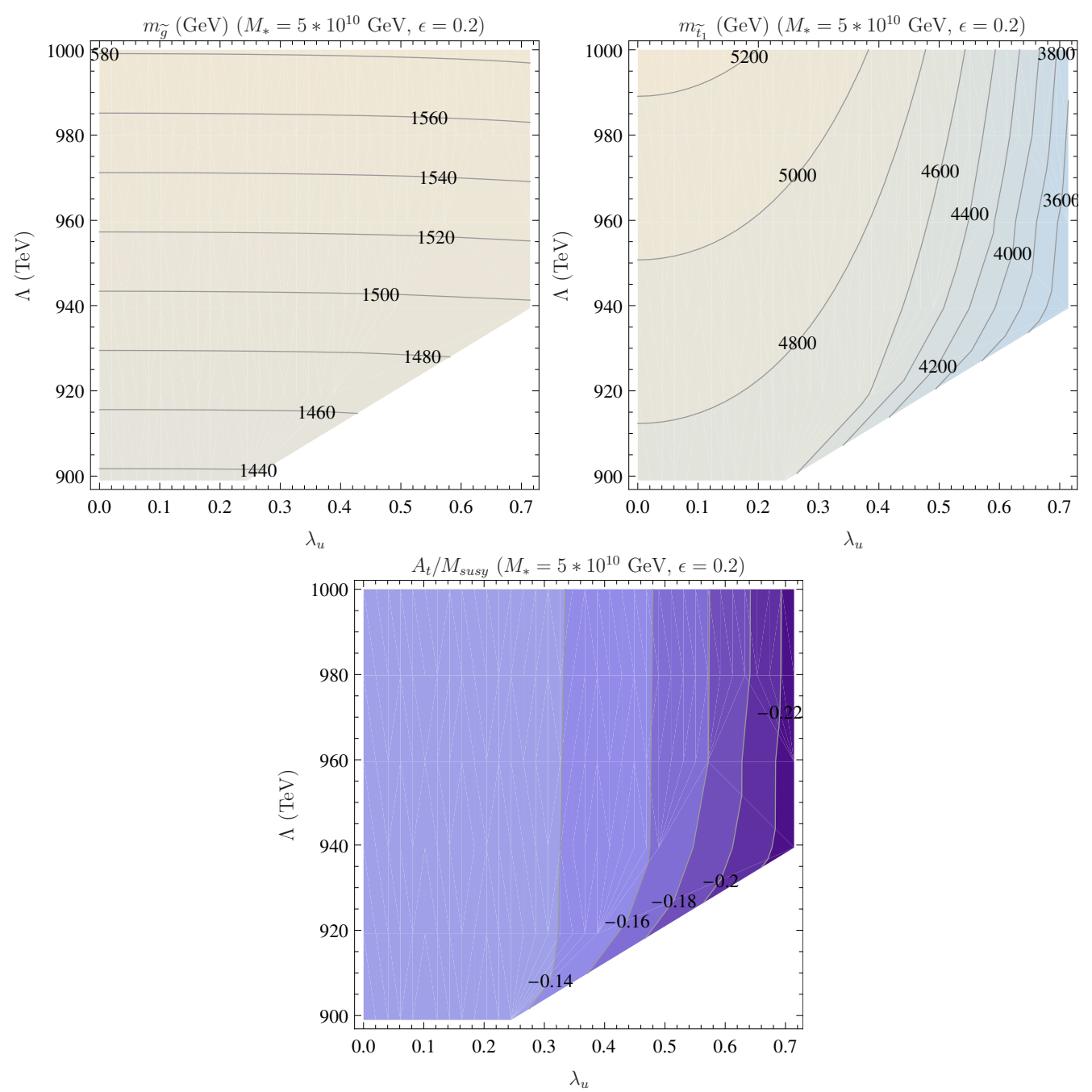

Figure 7. Similar to figure 6 , but for $M_{*}=5 \times 10^{10} \mathrm{GeV}, \epsilon=0.2$.

is signficantly smaller than in minimal GMSB. To demonstrate this we performed a full fledged numerical calculation of the fine tuning in this model using the combination of the public tools SARAH and SPheno.

In this paper, we concentrated on a moderate value for the conformal scale, i.e., $M_{*}<$ $10^{10} \mathrm{GeV}$. There are numerous avenues for exploring models with high conformal scale. In particular, when $M_{*}>10^{10} \mathrm{GeV}$, the LSP is no longer the Gravitino but the lightest neutralino. Hence, we would have a standard WIMP candidate for dark matter as well.

\section{Acknowledgments}

TL was supported in part by the Natural Science Foundation of China under grant numbers 10821504, 11075194, 11135003, 11275246, and by the the National Basic Research Program of China (973 Program) under Grant No. 2010CB833000. FS is supported by the BMBF PT DESY Verbundprojekt 05H2013-THEORIE 'Vergleich von LHC-Daten mit supersymmetrischen Modellen'. 

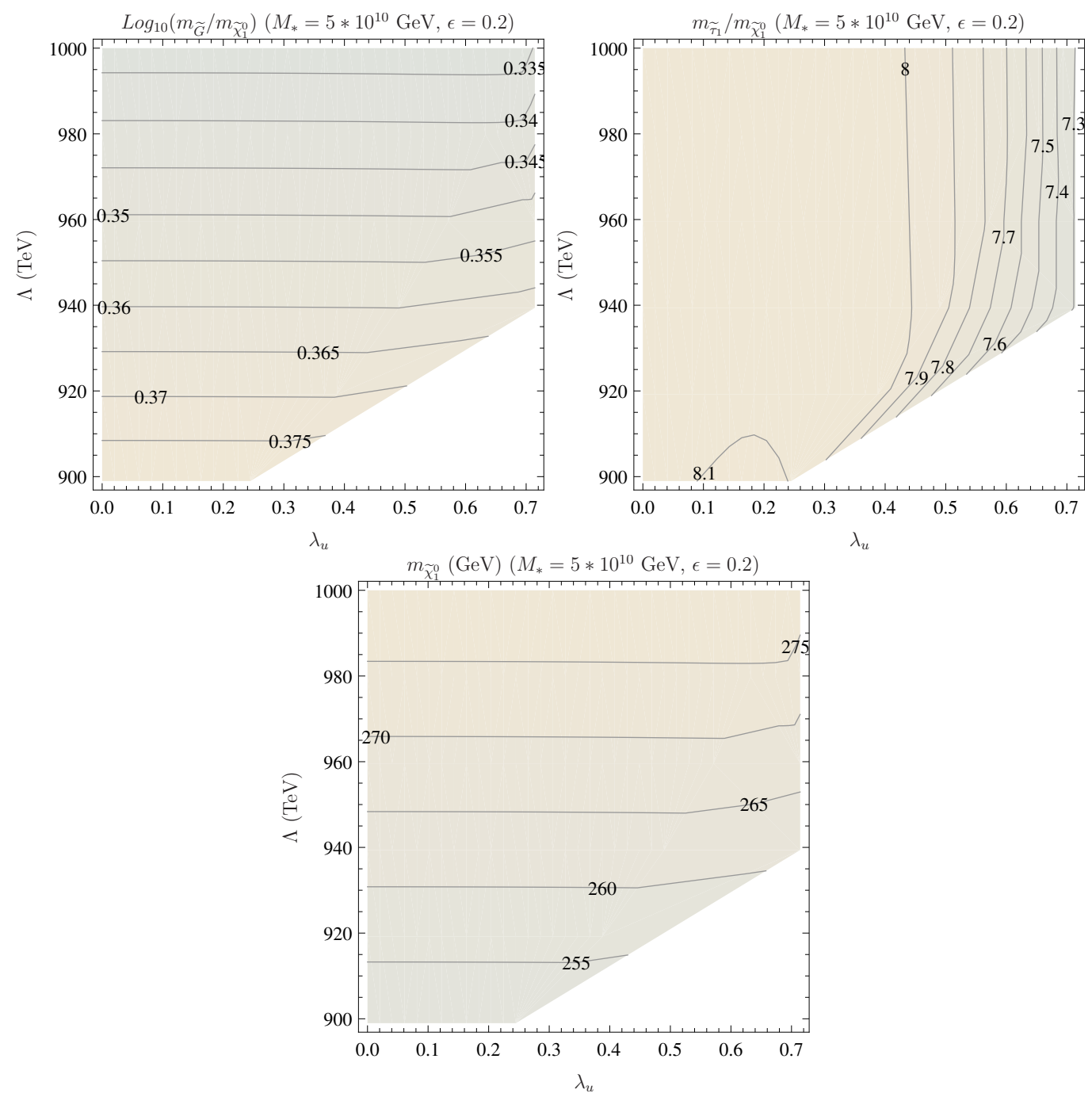

Figure 8. Mass ratio of the light stau and the neutralino $\left(m_{\tilde{\tau}_{1}} / m_{\tilde{\chi}_{1}^{0}}\right.$, on the top-left $)$, the mass ratio of the gravitino and neutralino $\left(\log _{10} m_{\tilde{G}} / m_{\tilde{\chi}_{1}^{0}}\right.$, top-right $)$ and absolute value of the neutralino mass $\left(m_{\tilde{\chi}_{1}^{0}}\right.$, bottom $)$ in the $\left(\lambda_{u}, \Lambda_{a}\right)$ plane. The other parameters are fixed to $M_{*}=5 \times 10^{10} \mathrm{GeV}$, $\epsilon=0.2, \tan \beta=10, n_{5}=1, n_{10}=0$, and $\operatorname{sign}(\mu)=1$.

\section{A Conventions and one-loop RGEs}

Our convention is the same as that in refs. $[152,153]$. We define

$$
t=2 \log \left(\frac{M_{*}}{Q}\right),
$$

where $M_{*}$ is the conformal scale scale at which the hidden sector renormalization decouples. To simplify the analytical calculation, we only consider the third generation Yukawa 


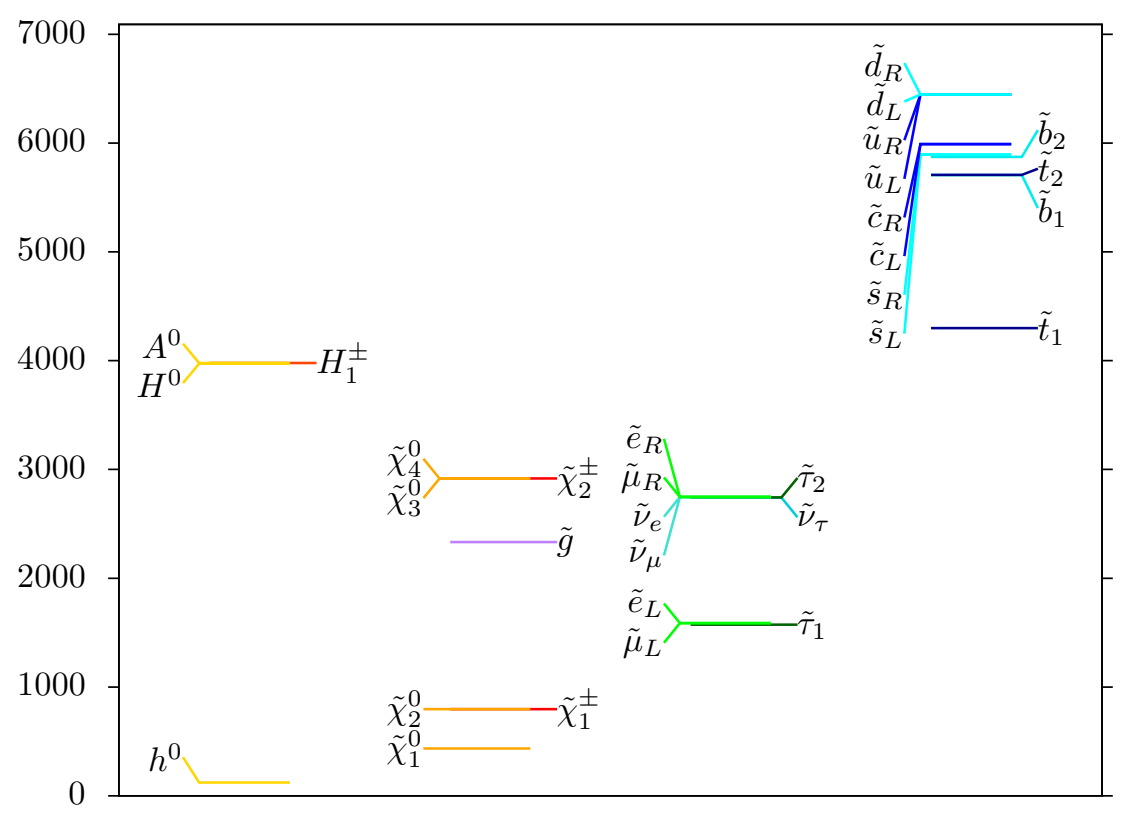

Figure 9. Mass Spectrum in the Best Point, in this case the LSP is still gravitino as in conventional GMSB model.

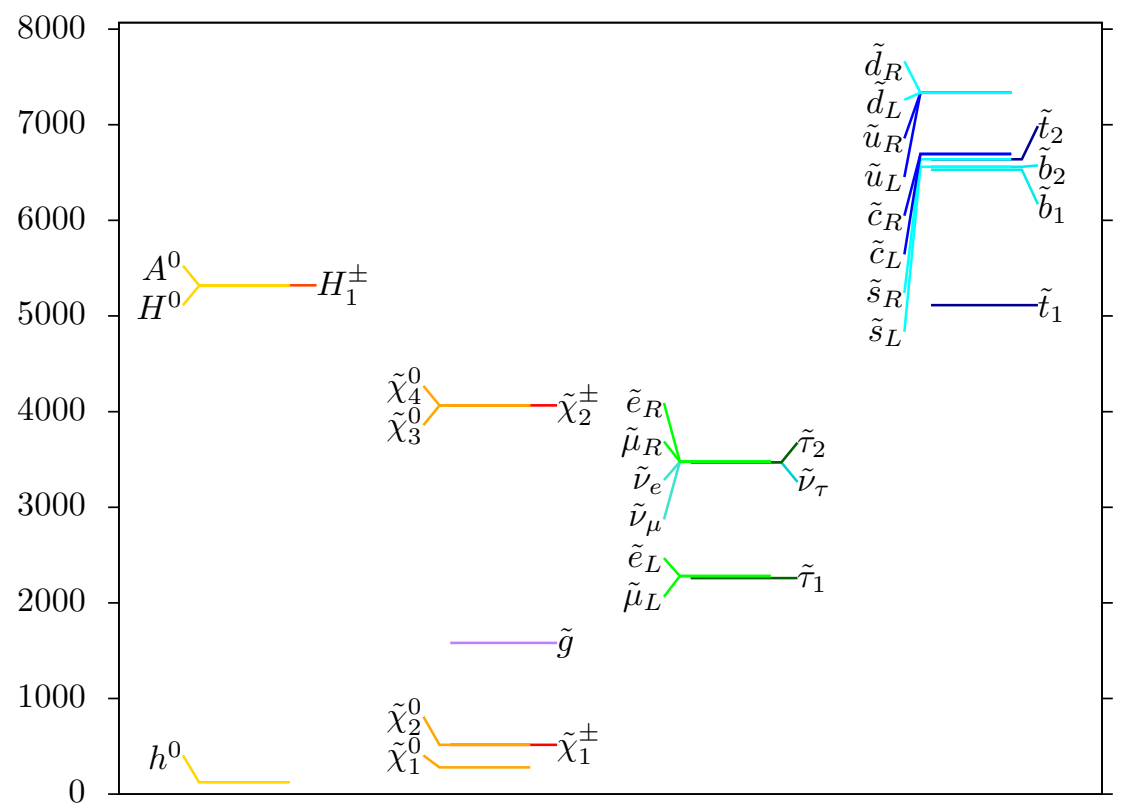

Figure 10. Similar to figure 9, but for conformal scale $M_{*}=5 \times 10^{10} \mathrm{GeV}$, in this case the LSP is neutralino. The other parameters are fixed to $\Lambda_{a}=10^{6} \mathrm{GeV}, \epsilon=0.2$ and $\lambda_{u}=0.3$. 
couplings and use the notation

$$
\begin{aligned}
\mathrm{a}_{i} & \equiv \frac{g_{i}^{2}}{16 \pi^{2}},(i=1,2,3), \\
Y_{k} & \equiv \frac{\left(y_{33}^{k}\right)^{2}}{16 \pi^{2}},(k=t, b, \tau), \\
\alpha_{\lambda} & \equiv \frac{\lambda_{u}^{2}}{16 \pi^{2}},
\end{aligned}
$$

where $\lambda$ is the marginal coupling between $H_{u}$ and messengers. The corresponding one-loop RGEs for the MSSM are

$$
\begin{aligned}
\frac{d \mathrm{a}_{i}}{d t} & =-b_{i} \mathrm{a}_{i}^{2}, \\
\frac{d Y_{t}}{d t} & =Y_{\tau}\left(\frac{16}{3} \mathrm{a}_{3}+3 \mathrm{a}_{2}+\frac{13}{15} \mathrm{a}_{1}-6 Y_{t}-Y_{b}\right), \\
\frac{d Y_{b}}{d t} & =Y_{b}\left(\frac{16}{3} \mathrm{a}_{3}+3 \mathrm{a}_{2}+\frac{7}{15} \mathrm{a}_{1}-Y_{t}-6 Y_{b}-Y_{\tau}\right), \\
\frac{d Y_{\tau}}{d t} & =Y_{\tau}\left(3 \mathrm{a}_{2}+\frac{9}{5} \mathrm{a}_{1}-3 Y_{b}-4 Y_{\tau}\right) .
\end{aligned}
$$

with

$$
\left(b_{1}, b_{2}, b_{3}\right)=(33 / 5,1,-3)
$$

The $\beta$-functions for the soft terms are given at one-loop by

$$
\begin{aligned}
\frac{d M_{i}}{d t}= & -b_{i} \mathrm{a}_{i} M_{i} \\
\frac{d A_{t}}{d t}= & -\left(\frac{16}{3} \mathrm{a}_{3} M_{3}+3 \mathrm{a}_{2} M_{2}+\frac{13}{15} \mathrm{a}_{1} M_{1}+6 Y_{t} A_{t}+Y_{b} A_{b}\right) \\
\frac{d A_{b}}{d t}= & -\left(\frac{16}{3} \mathrm{a}_{3} M_{3}+3 \mathrm{a}_{2} M_{2}+\frac{7}{15} \mathrm{a}_{1} M_{1}+6 Y_{b} A_{b}+Y_{t} A_{t}+Y_{\tau} A_{\tau}\right) \\
\frac{d A_{\tau}}{d t}= & -\left(3 \mathrm{a}_{2} M_{2}+\frac{9}{5} \mathrm{a}_{1} M_{1}+3 Y_{b} A_{b}+4 Y_{\tau} A_{\tau}\right) \\
\frac{d B}{d t}= & -\left(3 \mathrm{a}_{2} M_{2}+\frac{3}{5} \mathrm{a}_{1} M_{1}+3 Y_{t} A_{t}+3 Y_{b} A_{b}+Y_{\tau} A_{\tau}\right) \\
\frac{d \tilde{m}_{Q}^{2}}{d t}= & \left(\frac{16}{3} \mathrm{a}_{3} M_{3}^{2}+3 \mathrm{a}_{2} M_{2}^{2}+\frac{1}{15} \mathrm{a}_{1} M_{1}^{2}\right)-Y_{t}\left(\tilde{m}_{Q}^{2}+\tilde{m}_{U}^{2}+m_{H_{u}}^{2}+A_{t}^{2}\right) \\
& -Y_{b}\left(\tilde{m}_{Q}^{2}+\tilde{m}_{D}^{2}+m_{H_{d}}^{2}+A_{b}^{2}\right), \\
\frac{d \tilde{m}_{U}^{2}}{d t}= & \left(\frac{16}{3} \mathrm{a}_{3} M_{3}^{2}+\frac{16}{15} \mathrm{a}_{1} M_{1}^{2}\right)-2 Y_{t}\left(\tilde{m}_{Q}^{2}+\tilde{m}_{U}^{2}+m_{H_{u}}^{2}+A_{t}^{2}\right), \\
\frac{d \tilde{m}_{D}^{2}}{d t}= & \left(\frac{16}{3} \mathrm{a}_{3} M_{3}^{2}+\frac{4}{15} \mathrm{a}_{1} M_{1}^{2}\right)-2 Y_{b}\left(\tilde{m}_{Q}^{2}+\tilde{m}_{D}^{2}+m_{H_{d}}^{2}+A_{b}^{2}\right), \\
\frac{d \tilde{m}_{L}^{2}}{d t}= & 3\left(\mathrm{a}_{2} M_{2}^{2}+\frac{1}{5} \mathrm{a}_{1} M_{1}^{2}\right)-Y_{\tau}\left(\tilde{m}_{L}^{2}+\tilde{m}_{E}^{2}+m_{H_{d}}^{2}+A_{\tau}^{2}\right),
\end{aligned}
$$




$$
\begin{aligned}
\frac{d \tilde{m}_{E}^{2}}{d t}= & \left(\frac{12}{5} \mathrm{a}_{1} M_{1}^{2}\right)-2 Y_{\tau}\left(\tilde{m}_{L}^{2}+\tilde{m}_{E}^{2}+m_{H_{d}}^{2}+A_{\tau}^{2}\right), \\
\frac{d \mu^{2}}{d t}= & \mu^{2}\left[3\left(\mathrm{a}_{2}+\frac{1}{5} \mathrm{a}_{1}\right)-\left(3 Y_{t}+3 Y_{b}+Y_{\tau}\right)\right] \\
\frac{d m_{H_{d}}^{2}}{d t}= & 3\left(\mathrm{a}_{2} M_{2}^{2}+\frac{1}{5} \mathrm{a}_{1} M_{1}^{2}\right)-3 Y_{b}\left(\tilde{m}_{Q}^{2}+\tilde{m}_{D}^{2}+m_{H_{d}}^{2}+A_{b}^{2}\right) \\
& -Y_{\tau}\left(\tilde{m}_{L}^{2}+\tilde{m}_{E}^{2}+m_{H_{d}}^{2}+A_{\tau}^{2}\right), \\
\frac{d m_{H_{u}}^{2}}{d t}= & 3\left(\mathrm{a}_{2} M_{2}^{2}+\frac{1}{5} \mathrm{a}_{1} M_{1}^{2}\right)-3 Y_{t}\left(\tilde{m}_{Q}^{2}+\tilde{m}_{U}^{2}+m_{H_{u}}^{2}+A_{t}^{2}\right),
\end{aligned}
$$

\section{B General derivation of focus point formula}

In this appendix, we reproduce the well-known formula for focus point SUSY proposed in refs. $[27,29,76]$ in the context of our conventions of section A. In the region of small $\tan \beta$, the RGEs can be solved analytically [154]

$$
\begin{aligned}
\mathrm{a}_{i}[t] & =\frac{\mathrm{a}_{i}[0]}{1+\mathrm{a}_{i}[0] b_{i} t}, \\
Y_{t}[t] & =\frac{Y_{t}[0] E[t]}{\left.1+6 Y_{t}[0] F_{[} t\right]},
\end{aligned}
$$

where

$$
\begin{aligned}
E[t] & =\prod_{i}\left(1+b_{i} \mathrm{a}_{i}[0] t\right)^{c_{i} / b_{i}}, \\
c_{i} & =\left(\frac{13}{15}, 3, \frac{16}{3}\right), \\
F[t] & =\int_{0}^{t} E\left[t^{\prime}\right] d t^{\prime} .
\end{aligned}
$$

Therefore, we have

$$
\begin{aligned}
\mathcal{I} & =\exp \left(-6 \int_{0}^{t} Y_{t}\left[t^{\prime}\right] d t^{\prime}\right) \\
& =\exp \left(-6 \int_{0}^{t} \frac{Y_{t}[0] E\left[t^{\prime}\right]}{1+6 Y_{t}[0] F\left[t^{\prime}\right]} d t^{\prime}\right) \\
& =\frac{1}{1+6 Y_{t}[0] F[t]} \\
& =1-\frac{6 Y_{t}[t] F[t]}{E[t]}
\end{aligned}
$$

The formula eq. (B.6) plays a crucial role in determining whether or not focus point supersymmetry is available in a given model. Note, compared to ref. [29] there is an opposite sign. The reason is the different definition of running parameter in eq. (A.1).

Open Access. This article is distributed under the terms of the Creative Commons Attribution License (CC-BY 4.0), which permits any use, distribution and reproduction in any medium, provided the original author(s) and source are credited. 


\section{References}

[1] ATLAS collaboration, Observation of a new particle in the search for the Standard Model Higgs boson with the ATLAS detector at the LHC, Phys. Lett. B 716 (2012) 1 [arXiv:1207.7214] [INSPIRE].

[2] CMS collaboration, Observation of a new boson at a mass of $125 \mathrm{GeV}$ with the CMS experiment at the LHC, Phys. Lett. B 716 (2012) 30 [arXiv:1207.7235] [InSPIRE].

[3] M.S. Carena and H.E. Haber, Higgs boson theory and phenomenology, Prog. Part. Nucl. Phys. 50 (2003) 63 [hep-ph/0208209] [INSPIRE].

[4] A. Djouadi, The anatomy of electro-weak symmetry breaking. II. The Higgs bosons in the minimal supersymmetric model, Phys. Rept. 459 (2008) 1 [hep-ph/0503173] [InSPIRE].

[5] M.S. Carena, P.H. Chankowski, S. Pokorski and C. Wagner, The Higgs boson mass as a probe of the minimal supersymmetric standard model, Phys. Lett. B 441 (1998) 205 [hep-ph/9805349] [INSPIRE].

[6] A. Djouadi, Implications of the Higgs discovery for the MSSM, arXiv:1311.0720 [INSPIRE].

[7] M. Carena, S. Heinemeyer, O. Stål, C. Wagner and G. Weiglein, MSSM Higgs Boson Searches at the LHC: Benchmark Scenarios after the Discovery of a Higgs-like Particle, Eur. Phys. J. C 73 (2013) 2552 [arXiv:1302.7033] [INSPIRE].

[8] G. Degrassi, P. Slavich and F. Zwirner, On the neutral Higgs boson masses in the MSSM for arbitrary stop mixing, Nucl. Phys. B 611 (2001) 403 [hep-ph/0105096] [INSPIRE].

[9] A. Brignole, G. Degrassi, P. Slavich and F. Zwirner, On the $O\left(\alpha(t)^{2}\right)$ two loop corrections to the neutral Higgs boson masses in the MSSM, Nucl. Phys. B 631 (2002) 195 [hep-ph/0112177] [INSPIRE].

[10] A. Brignole, G. Degrassi, P. Slavich and F. Zwirner, On the two loop sbottom corrections to the neutral Higgs boson masses in the MSSM, Nucl. Phys. B 643 (2002) 79 [hep-ph/0206101] [INSPIRE].

[11] A. Dedes and P. Slavich, Two loop corrections to radiative electroweak symmetry breaking in the MSSM, Nucl. Phys. B 657 (2003) 333 [hep-ph/0212132] [INSPIRE].

[12] A. Dedes, G. Degrassi and P. Slavich, On the two loop Yukawa corrections to the MSSM Higgs boson masses at large tan beta, Nucl. Phys. B 672 (2003) 144 [hep-ph/0305127] [INSPIRE].

[13] P. Kant, Three-Loop Predictions for the Light Higgs Mass in the MSSM, arXiv:1008.0239 [INSPIRE].

[14] J. Camargo-Molina, B. O'Leary, W. Porod and F. Staub, Stability of the CMSSM against sfermion VEVs, JHEP 12 (2013) 103 [arXiv:1309.7212] [INSPIRE].

[15] N. Blinov and D.E. Morrissey, Vacuum Stability and the MSSM Higgs Mass, arXiv: 1310.4174 [INSPIRE].

[16] D. Chowdhury, R.M. Godbole, K.A. Mohan and S.K. Vempati, Charge and Color Breaking Constraints in MSSM after the Higgs Discovery at LHC, arXiv:1310.1932 [INSPIRE].

[17] R. Barbieri and G. Giudice, Upper Bounds on Supersymmetric Particle Masses, Nucl. Phys. B 306 (1988) 63 [inSPIRE].

[18] G.W. Anderson and D.J. Castano, Measures of fine tuning, Phys. Lett. B 347 (1995) 300 [hep-ph/9409419] [INSPIRE]. 
[19] A.G. Cohen, D. Kaplan and A. Nelson, The more minimal supersymmetric standard model, Phys. Lett. B 388 (1996) 588 [hep-ph/9607394] [INSPIRE].

[20] P. Ciafaloni and A. Strumia, Naturalness upper bounds on gauge mediated soft terms, Nucl. Phys. B 494 (1997) 41 [hep-ph/9611204] [inSPIRE].

[21] G. Bhattacharyya and A. Romanino, Naturalness constraints on gauge mediated supersymmetry breaking models, Phys. Rev. D 55 (1997) 7015 [hep-ph/9611243] [INSPIRE].

[22] P.H. Chankowski, J.R. Ellis and S. Pokorski, The fine tuning price of LEP, Phys. Lett. B 423 (1998) 327 [hep-ph/9712234] [INSPIRE].

[23] R. Barbieri and A. Strumia, About the fine tuning price of LEP, Phys. Lett. B 433 (1998) 63 [hep-ph/9801353] [INSPIRE].

[24] G.L. Kane and S. King, Naturalness implications of LEP results, Phys. Lett. B 451 (1999) 113 [hep-ph/9810374] [INSPIRE].

[25] L. Giusti, A. Romanino and A. Strumia, Natural ranges of supersymmetric signals, Nucl. Phys. B 550 (1999) 3 [hep-ph/9811386] [INSPIRE].

[26] M. Bastero-Gil, G.L. Kane and S. King, Fine tuning constraints on supergravity models, Phys. Lett. B 474 (2000) 103 [hep-ph/9910506] [INSPIRE].

[27] J.L. Feng, K.T. Matchev and T. Moroi, Multi-TeV scalars are natural in minimal supergravity, Phys. Rev. Lett. 84 (2000) 2322 [hep-ph/9908309] [INSPIRE].

[28] A. Romanino and A. Strumia, Are heavy scalars natural in minimal supergravity?, Phys. Lett. B 487 (2000) 165 [hep-ph/9912301] [INSPIRE].

[29] J.L. Feng, K.T. Matchev and T. Moroi, Focus points and naturalness in supersymmetry, Phys. Rev. D 61 (2000) 075005 [hep-ph/9909334] [inSPIRE].

[30] Z. Chacko, Y. Nomura and D. Tucker-Smith, A minimally fine-tuned supersymmetric standard model, Nucl. Phys. B 725 (2005) 207 [hep-ph/0504095] [INSPIRE].

[31] K. Choi, K.S. Jeong, T. Kobayashi and K.-i. Okumura, Little SUSY hierarchy in mixed modulus-anomaly mediation, Phys. Lett. B 633 (2006) 355 [hep-ph/0508029] [INSPIRE].

[32] Y. Nomura and B. Tweedie, The supersymmetric fine-tuning problem and TeV-scale exotic scalars, Phys. Rev. D 72 (2005) 015006 [hep-ph/0504246] [INSPIRE].

[33] R. Kitano and Y. Nomura, A solution to the supersymmetric fine-tuning problem within the MSSM, Phys. Lett. B 631 (2005) 58 [hep-ph/0509039] [INSPIRE].

[34] Y. Nomura, D. Poland and B. Tweedie, Minimally fine-tuned supersymmetric standard models with intermediate-scale supersymmetry breaking, Nucl. Phys. B 745 (2006) 29 [hep-ph/0509243] [INSPIRE].

[35] O. Lebedev, H.P. Nilles and M. Ratz, A note on fine-tuning in mirage mediation, hep-ph/0511320 [INSPIRE].

[36] R. Kitano and Y. Nomura, Supersymmetry, naturalness and signatures at the LHC, Phys. Rev. D 73 (2006) 095004 [hep-ph/0602096] [INSPIRE].

[37] B. Allanach, Naturalness priors and fits to the constrained minimal supersymmetric standard model, Phys. Lett. B 635 (2006) 123 [hep-ph/0601089] [INSPIRE].

[38] G. Giudice and R. Rattazzi, Living Dangerously with Low-Energy Supersymmetry, Nucl. Phys. B 757 (2006) 19 [hep-ph/0606105] [INSPIRE]. 
[39] M. Perelstein and C. Spethmann, A collider signature of the supersymmetric golden region, JHEP 04 (2007) 070 [hep-ph/0702038] [INSPIRE].

[40] B.C. Allanach, K. Cranmer, C.G. Lester and A.M. Weber, Natural priors, CMSSM fits and LHC weather forecasts, JHEP 08 (2007) 023 [arXiv: 0705.0487] [INSPIRE].

[41] M. Cabrera, J. Casas and R. Ruiz de Austri, Bayesian approach and naturalness in MSSM analyses for the LHC, JHEP 03 (2009) 075 [arXiv:0812.0536] [INSPIRE].

[42] S. Cassel, D. Ghilencea and G. Ross, Fine tuning as an indication of physics beyond the MSSM, Nucl. Phys. B 825 (2010) 203 [arXiv:0903.1115] [InSPIRE].

[43] R. Barbieri and D. Pappadopulo, S-particles at their naturalness limits, JHEP 10 (2009) 061 [arXiv: 0906.4546] [INSPIRE].

[44] D. Horton and G. Ross, Naturalness and Focus Points with Non-Universal Gaugino Masses, Nucl. Phys. B 830 (2010) 221 [arXiv:0908.0857] [inSPIRE].

[45] T. Kobayashi, Y. Nakai and R. Takahashi, Fine tuning in general gauge mediation, JHEP 01 (2010) 003 [arXiv: 0910.3477] [INSPIRE].

[46] P. Lodone, Naturalness bounds in extensions of the MSSM without a light Higgs boson, JHEP 05 (2010) 068 [arXiv:1004.1271] [INSPIRE].

[47] M. Asano, H.D. Kim, R. Kitano and Y. Shimizu, Natural supersymmetry at the LHC, JHEP 12 (2010) 019 [arXiv:1010.0692] [InSPIRE].

[48] A. Strumia, The fine-tuning price of the early LHC, JHEP 04 (2011) 073 [arXiv: 1101.2195] [INSPIRE].

[49] S. Cassel, D. Ghilencea, S. Kraml, A. Lessa and G. Ross, Fine-tuning implications for complementary dark matter and LHC SUSY searches, JHEP 05 (2011) 120 [arXiv: 1101.4664] [INSPIRE].

[50] K. Sakurai and K. Takayama, Constraint from recent ATLAS results to non-universal sfermion mass models and naturalness, JHEP 12 (2011) 063 [arXiv:1106.3794] [InSPIRE].

[51] M. Papucci, J.T. Ruderman and A. Weiler, Natural SUSY endures, JHEP 09 (2012) 035 [arXiv: 1110.6926$]$ [INSPIRE].

[52] G. Larsen, Y. Nomura and H.L. Roberts, Supersymmetry with light stops, JHEP 06 (2012) 032 [arXiv: 1202.6339] [INSPIRE].

[53] H. Baer, V. Barger, P. Huang and X. Tata, Natural supersymmetry: LHC, dark matter and ILC searches, JHEP 05 (2012) 109 [arXiv:1203.5539] [INSPIRE].

[54] J.R. Espinosa, C. Grojean, V. Sanz and M. Trott, NSUSY fits, JHEP 12 (2012) 077 [arXiv:1207.7355] [INSPIRE].

[55] J.R. Ellis, K. Enqvist, D.V. Nanopoulos and F. Zwirner, Observables in Low-Energy Superstring Models, Mod. Phys. Lett. A 1 (1986) 57 [INSPIRE].

[56] N. Ohta, Grand unified theories based on local supersymmetry, Prog. Theor. Phys. 70 (1983) 542 [INSPIRE].

[57] H. Baer et al., Post-LHC7 fine-tuning in the mSUGRA/CMSSM model with a $125 \mathrm{GeV}$ Higgs boson, Phys. Rev. D 87 (2013) 035017 [arXiv:1210.3019] [INSPIRE].

[58] U. Ellwanger, C. Hugonie and A.M. Teixeira, The Next-to-Minimal Supersymmetric Standard Model, Phys. Rept. 496 (2010) 1 [arXiv:0910.1785] [INSPIRE]. 
[59] U. Ellwanger and C. Hugonie, The upper bound on the lightest Higgs mass in the NMSSM revisited, Mod. Phys. Lett. A 22 (2007) 1581 [hep-ph/0612133] [INSPIRE].

[60] E. Ma, Exceeding the MSSM Higgs Mass Bound in a Special Class of U(1) Gauge Models, Phys. Lett. B 705 (2011) 320 [arXiv:1108.4029] [INSPIRE].

[61] Y. Zhang, H. An, X.-d. Ji and R.N. Mohapatra, Light Higgs Mass Bound in SUSY Left-Right Models, Phys. Rev. D 78 (2008) 011302 [arXiv: 0804.0268] [inSPIRE].

[62] M. Hirsch, M. Malinsky, W. Porod, L. Reichert and F. Staub, Hefty MSSM-like light Higgs in extended gauge models, JHEP 02 (2012) 084 [arXiv:1110.3037] [INSPIRE].

[63] A. Bharucha, A. Goudelis and M. McGarrie, En-gauging naturalness, arXiv:1310.4500 [INSPIRE].

[64] M. Bastero-Gil, C. Hugonie, S. King, D. Roy and S. Vempati, Does LEP prefer the NMSSM?, Phys. Lett. B 489 (2000) 359 [hep-ph/0006198] [inSPIRE].

[65] R. Dermisek and J.F. Gunion, Consistency of LEP event excesses with an $h \rightarrow$ aa decay scenario and low-fine-tuning NMSSM models, Phys. Rev. D 73 (2006) 111701 [hep-ph/0510322] [INSPIRE].

[66] R. Dermisek, J.F. Gunion and B. McElrath, Probing NMSSM Scenarios with Minimal Fine-Tuning by Searching for Decays of the Upsilon to a Light CP-Odd Higgs Boson, Phys. Rev. D 76 (2007) 051105 [hep-ph/0612031] [INSPIRE].

[67] R. Dermisek and J.F. Gunion, The NMSSM Solution to the Fine-Tuning Problem, Precision Electroweak Constraints and the Largest LEP Higgs Event Excess, Phys. Rev. D 76 (2007) 095006 [arXiv: 0705.4387] [INSPIRE].

[68] U. Ellwanger, G. Espitalier-Noel and C. Hugonie, Naturalness and Fine Tuning in the NMSSM: Implications of Early LHC Results, JHEP 09 (2011) 105 [arXiv:1107.2472] [INSPIRE].

[69] H.M. Lee et al., A unique $Z_{4}^{R}$ symmetry for the MSSM, Phys. Lett. B 694 (2011) 491 [arXiv: 1009.0905] [INSPIRE].

[70] H.M. Lee et al., Discrete $R$ symmetries for the MSSM and its singlet extensions, Nucl. Phys. B 850 (2011) 1 [arXiv:1102.3595] [INSPIRE].

[71] G.G. Ross and K. Schmidt-Hoberg, The Fine-Tuning of the Generalised NMSSM, Nucl. Phys. B 862 (2012) 710 [arXiv:1108.1284] [INSPIRE].

[72] G.G. Ross, K. Schmidt-Hoberg and F. Staub, The generalised NMSSM at one loop: fine tuning and phenomenology, JHEP 08 (2012) 074 [arXiv: 1205.1509] [INSPIRE].

[73] A. Kaminska, G.G. Ross and K. Schmidt-Hoberg, Non-universal gaugino masses and fine tuning implications for SUSY searches in the MSSM and the GNMSSM, JHEP 11 (2013) 209 [arXiv: 1308.4168] [INSPIRE].

[74] X. Lu, H. Murayama, J.T. Ruderman and K. Tobioka, A Natural Higgs Mass in Supersymmetry from Non-Decoupling Effects, arXiv:1308.0792 [INSPIRE].

[75] A. Kaminska, G.G. Ross, K. Schmidt-Hoberg and F. Staub, A precision study of the fine tuning in the DiracNMSSM, arXiv:1401.1816 [INSPIRE].

[76] J.L. Feng and K.T. Matchev, Focus point supersymmetry: Proton decay, flavor and CP-violation and the Higgs boson mass, Phys. Rev. D 63 (2001) 095003 [hep-ph/0011356] [INSPIRE]. 
[77] J.L. Feng, K.T. Matchev and D. Sanford, Focus Point Supersymmetry Redux, Phys. Rev. D 85 (2012) 075007 [arXiv: 1112.3021] [InSPIRE].

[78] J.L. Feng and D. Sanford, A Natural 125 GeV Higgs Boson in the MSSM from Focus Point Supersymmetry with A-Terms, Phys. Rev. D 86 (2012) 055015 [arXiv:1205.2372] [INSPIRE].

[79] K. Agashe, Can multi-TeV (top and other) squarks be natural in gauge mediation?, Phys. Rev. D 61 (2000) 115006 [hep-ph/9910497] [INSPIRE].

[80] F. Brümmer, M. Ibe and T.T. Yanagida, Focus point gauge mediation in product group unification, Phys. Lett. B 726 (2013) 364 [arXiv:1303.1622] [INSPIRE].

[81] S. Zheng, Focus Point from Direct Gauge Mediation, arXiv:1312.4105 [INSPIRE].

[82] H. Abe, T. Kobayashi and Y. Omura, Relaxed fine-tuning in models with non-universal gaugino masses, Phys. Rev. D 76 (2007) 015002 [hep-ph/0703044] [INSPIRE].

[83] J.E. Younkin and S.P. Martin, Non-universal gaugino masses, the supersymmetric little hierarchy problem and dark matter, Phys. Rev. D 85 (2012) 055028 [arXiv:1201.2989] [INSPIRE].

[84] T.T. Yanagida and N. Yokozaki, Focus Point in Gaugino Mediation Reconsideration of the Fine-tuning Problem , Phys. Lett. B 722 (2013) 355 [arXiv:1301.1137] [InSPIRE].

[85] K.L. Chan, U. Chattopadhyay and P. Nath, Naturalness, weak scale supersymmetry and the prospect for the observation of supersymmetry at the Tevatron and at the CERN LHC, Phys. Rev. D 58 (1998) 096004 [hep-ph/9710473] [INSPIRE].

[86] S. Akula, M. Liu, P. Nath and G. Peim, Naturalness, Supersymmetry and Implications for LHC and Dark Matter, Phys. Lett. B 709 (2012) 192 [arXiv:1111.4589] [InSPIRE].

[87] M. Dine and W. Fischler, A Phenomenological Model of Particle Physics Based on Supersymmetry, Phys. Lett. B 110 (1982) 227 [InSPIRE].

[88] M. Dine, W. Fischler and M. Srednicki, Supersymmetric Technicolor, Nucl. Phys. B 189 (1981) 575 [INSPIRE].

[89] S. Dimopoulos and S. Raby, Supercolor, Nucl. Phys. B 192 (1981) 353 [inSPIRE].

[90] C.R. Nappi and B.A. Ovrut, Supersymmetric Extension of the $\mathrm{SU}(3) \times \mathrm{SU}(2) \times \mathrm{U}(1)$ Model, Phys. Lett. B 113 (1982) 175 [INSPIRE].

[91] L. Álvarez-Gaumé, M. Claudson and M.B. Wise, Low-Energy Supersymmetry, Nucl. Phys. B 207 (1982) 96 [INSPIRE].

[92] M. Dine and A.E. Nelson, Dynamical supersymmetry breaking at low-energies, Phys. Rev. D 48 (1993) 1277 [hep-ph/9303230] [INSPIRE].

[93] M. Dine, R.G. Leigh and A. Kagan, Supersymmetry and the Nelson-Barr mechanism, Phys. Rev. D 48 (1993) 2214 [hep-ph/9303296] [INSPIRE].

[94] M. Dine, A.E. Nelson and Y. Shirman, Low-energy dynamical supersymmetry breaking simplified, Phys. Rev. D 51 (1995) 1362 [hep-ph/9408384] [INSPIRE].

[95] M. Dine, A.E. Nelson, Y. Nir and Y. Shirman, New tools for low-energy dynamical supersymmetry breaking, Phys. Rev. D 53 (1996) 2658 [hep-ph/9507378] [INSPIRE].

[96] S. Raby, Gauge mediated SUSY breaking at an intermediate scale, Phys. Rev. D 56 (1997) 2852 [hep-ph/9702299] [INSPIRE]. 
[97] P. Draper, P. Meade, M. Reece and D. Shih, Implications of a 125 GeV Higgs for the MSSM and Low-Scale SUSY Breaking, Phys. Rev. D 85 (2012) 095007 [arXiv:1112.3068] [INSPIRE].

[98] M.E. Krauss, W. Porod and F. Staub, $\mathrm{SO}(10)$ inspired gauge-mediated supersymmetry breaking, Phys. Rev. D 88 (2013) 015014 [arXiv: 1304.0769] [InSPIRE].

[99] Y. Kahn, M. McCullough and J. Thaler, Auxiliary gauge mediation: a new route to mini-split supersymmetry, JHEP 11 (2013) 161 [arXiv:1308.3490] [INSPIRE].

[100] Y. Shadmi and P.Z. Szabo, Flavored gauge-mediation, JHEP 06 (2012) 124 [arXiv:1103.0292] [INSPIRE].

[101] J.L. Evans, M. Ibe and T.T. Yanagida, Relatively Heavy Higgs Boson in More Generic Gauge Mediation, Phys. Lett. B 705 (2011) 342 [arXiv:1107.3006] [InSPIRE].

[102] T. Jelinski, J. Pawełczyk and K. Turzynski, On Low-Energy Predictions of Unification Models Inspired by F-theory, Phys. Lett. B 711 (2012) 307 [arXiv:1111.6492] [INSPIRE].

[103] J.L. Evans, M. Ibe, S. Shirai and T.T. Yanagida, A 125GeV Higgs Boson and Muon g-2 in More Generic Gauge Mediation, Phys. Rev. D 85 (2012) 095004 [arXiv:1201.2611] [INSPIRE].

[104] A. Albaid and K. Babu, Higgs boson of mass $125 \mathrm{GeV}$ in GMSB models with messenger-matter mixing, Phys. Rev. D 88 (2013) 055007 [arXiv:1207.1014] [INSPIRE].

[105] M. Abdullah, I. Galon, Y. Shadmi and Y. Shirman, Flavored Gauge Mediation, A Heavy Higgs and Supersymmetric Alignment, JHEP 06 (2013) 057 [arXiv: 1209.4904] [InSPIRE].

[106] M.J. Perez, P. Ramond and J. Zhang, Mixing supersymmetry and family symmetry breakings, Phys. Rev. D 87 (2013) 035021 [arXiv:1209.6071] [INSPIRE].

[107] M. Endo, K. Hamaguchi, S. Iwamoto and N. Yokozaki, Vacuum stability bound on extended GMSB models, JHEP 06 (2012) 060 [arXiv:1202.2751] [INSPIRE].

[108] G. Giudice and R. Rattazzi, Theories with gauge mediated supersymmetry breaking, Phys. Rept. 322 (1999) 419 [hep-ph/9801271] [INSPIRE].

[109] N. Craig, S. Knapen, D. Shih and Y. Zhao, A complete model of low-scale gauge mediation, JHEP 03 (2013) 154 [arXiv:1206.4086] [InSPIRE].

[110] Z. Kang, T. Li, T. Liu, C. Tong and J.M. Yang, A Heavy SM-like Higgs and a Light Stop from Yukawa-Deflected Gauge Mediation, Phys. Rev. D 86 (2012) 095020 [arXiv:1203.2336] [INSPIRE].

[111] P. Byakti and T.S. Ray, Burgeoning the Higgs mass to $125 \mathrm{GeV}$ through messenger-matter interactions in GMSB models, JHEP 05 (2013) 055 [arXiv:1301.7605] [INSPIRE].

[112] N. Craig, S. Knapen and D. Shih, General Messenger Higgs Mediation, JHEP 08 (2013) 118 [arXiv: 1302.2642] [INSPIRE].

[113] J.A. Evans and D. Shih, Surveying Extended GMSB Models with $m_{h}=125$ GeV, JHEP 08 (2013) 093 [arXiv: 1303.0228] [INSPIRE].

[114] L. Calibbi, P. Paradisi and R. Ziegler, Gauge mediation beyond minimal flavor violation, JHEP 06 (2013) 052 [arXiv: 1304.1453] [InSPIRE].

[115] T. Jeliński, On messengers couplings in extended GMSB models, JHEP 09 (2013) 107 [arXiv: 1305.6277] [INSPIRE].

[116] I. Galon, G. Perez and Y. Shadmi, Non-degenerate squarks from flavored gauge mediation, JHEP 09 (2013) 117 [arXiv:1306.6631] [INSPIRE]. 
[117] W. Fischler and W. Tangarife, Vector-like Fields, Messenger Mixing and the Higgs mass in Gauge Mediation, arXiv:1310.6369 [INSPIRE].

[118] S. Knapen and D. Shih, Higgs mediation with strong hidden sector dynamics, arXiv: 1311.7107 [INSPIRE].

[119] F. Brümmer, M. McGarrie and A. Weiler, Light third-generation squarks from flavour gauge messengers, arXiv:1312.0935 [INSPIRE].

[120] J.L. Feng, Naturalness and the status of supersymmetry, Ann. Rev. Nucl. Part. Sci. 63 (2013) 351 [arXiv: 1302.6587] [INSPIRE].

[121] N. Craig, The State of Supersymmetry after Run I of the LHC, arXiv:1309.0528 [INSPIRE].

[122] A.G. Cohen, T.S. Roy and M. Schmaltz, Hidden sector renormalization of MSSM scalar masses, JHEP 02 (2007) 027 [hep-ph/0612100] [INSPIRE].

[123] T.S. Roy and M. Schmaltz, Hidden solution to the mu/Bmu problem in gauge mediation, Phys. Rev. D 77 (2008) 095008 [arXiv: 0708.3593] [INSPIRE].

[124] H. Murayama, Y. Nomura and D. Poland, More visible effects of the hidden sector, Phys. Rev. D 77 (2008) 015005 [arXiv: 0709.0775] [INSPIRE].

[125] S. Shirai, F. Takahashi, T. Yanagida and K. Yonekura, Gauge Mediation with Sequestered Supersymmetry Breaking, Phys. Rev. D 78 (2008) 075003 [arXiv: 0808.0848] [INSPIRE].

[126] M. Asano, J. Hisano, T. Okada and S. Sugiyama, A Realistic Extension of Gauge-Mediated SUSY-Breaking Model with Superconformal Hidden Sector, Phys. Lett. B 673 (2009) 146 [arXiv:0810.4606] [INSPIRE].

[127] G. Perez, T.S. Roy and M. Schmaltz, Phenomenology of SUSY with scalar sequestering, Phys. Rev. D 79 (2009) 095016 [arXiv:0811.3206] [inSPIRE].

[128] S.P. Martin, Generalized messengers of supersymmetry breaking and the sparticle mass spectrum, Phys. Rev. D 55 (1997) 3177 [hep-ph/9608224] [INSPIRE].

[129] D. Poland, D. Simmons-Duffin and A. Vichi, Carving out the space of $4 D$ CFTs, JHEP 05 (2012) 110 [arXiv:1109.5176] [INSPIRE].

[130] F. Staub, SARAH, arXiv:0806.0538 [INSPIRE].

[131] F. Staub, From Superpotential to Model Files for FeynArts and CalcHep/CompHEP, Comput. Phys. Commun. 181 (2010) 1077 [arXiv:0909.2863] [InSPIRE].

[132] F. Staub, Automatic Calculation of supersymmetric Renormalization Group Equations and Self Energies, Comput. Phys. Commun. 182 (2011) 808 [arXiv: 1002.0840] [InSPIRE].

[133] F. Staub, SARAH 3.2: Dirac Gauginos, UFO output and more, Comput. Phys. Commun. 184 (2013) 1792 [arXiv:1207.0906] [INSPIRE].

[134] F. Staub, SARAH 4: A tool for (not only SUSY) model builders, arXiv:1309.7223 [INSPIRE].

[135] W. Porod, SPheno, a program for calculating supersymmetric spectra, SUSY particle decays and SUSY particle production at $e^{+} e^{-}$colliders, Comput. Phys. Commun. 153 (2003) 275 [hep-ph/0301101] [INSPIRE].

[136] W. Porod and F. Staub, SPheno 3.1: Extensions including flavour, CP-phases and models beyond the MSSM, Comput. Phys. Commun. 183 (2012) 2458 [arXiv:1104.1573] [INSPIRE]. 
[137] ATLAS collaboration, Search for squarks and gluinos with the ATLAS detector in final states with jets and missing transverse momentum using $4.7 \mathrm{fb}^{-1}$ of $\sqrt{\mathrm{s}}=7 \mathrm{TeV}$ proton-proton collision data, Phys. Rev. D 87 (2013) 012008 [arXiv:1208.0949] [INSPIRE].

[138] CMS collaboration, Search for supersymmetry in hadronic final states using MT2 in pp collisions at $\sqrt{s}=7 \mathrm{TeV}$, JHEP 10 (2012) 018 [arXiv:1207.1798] [INSPIRE].

[139] F. Staub, T. Ohl, W. Porod and C. Speckner, A Tool Box for Implementing Supersymmetric Models, Comput. Phys. Commun. 183 (2012) 2165 [arXiv:1109.5147] [InSPIRE].

[140] N. Arkani-Hamed, S. Dimopoulos, G. Giudice and A. Romanino, Aspects of split supersymmetry, Nucl. Phys. B 709 (2005) 3 [hep-ph/0409232] [INSPIRE].

[141] G. Giudice and A. Romanino, Split supersymmetry, Nucl. Phys. B 699 (2004) 65 [Erratum ibid. B 706 (2005) 65-89] [hep-ph/0406088] [INSPIRE].

[142] N. Bernal, A. Djouadi and P. Slavich, The MSSM with heavy scalars, JHEP 07 (2007) 016 [arXiv:0705.1496] [INSPIRE].

[143] K. Harigaya, M. Ibe and T.T. Yanagida, A closer look at gaugino masses in pure gravity mediation model/minimal split SUSY model, JHEP 12 (2013) 016 [arXiv:1310.0643] [INSPIRE].

[144] D.S. Alves, E. Izaguirre and J.G. Wacker, Higgs, Binos and Gluinos: Split SUSY Within Reach, arXiv:1108.3390 [INSPIRE].

[145] T. Banks, D.B. Kaplan and A.E. Nelson, Cosmological implications of dynamical supersymmetry breaking, Phys. Rev. D 49 (1994) 779 [hep-ph/9308292] [INSPIRE].

[146] T. Moroi, H. Murayama and M. Yamaguchi, Cosmological constraints on the light stable gravitino, Phys. Lett. B 303 (1993) 289 [InSPIRE].

[147] L.J. Hall, J.D. Lykken and S. Weinberg, Supergravity as the Messenger of Supersymmetry Breaking, Phys. Rev. D 27 (1983) 2359 [InSPIRE].

[148] E.A. Baltz and H. Murayama, Gravitino warm dark matter with entropy production, JHEP 05 (2003) 067, [astro-ph/0108172] [INSPIRE].

[149] M. Kawasaki, F. Takahashi and T. Yanagida, Gravitino overproduction in inflaton decay, Phys. Lett. B 638 (2006) 8 [hep-ph/0603265] [INSPIRE].

[150] M. Kawasaki, K. Kohri, T. Moroi and A. Yotsuyanagi, Big-Bang Nucleosynthesis and Gravitino, Phys. Rev. D 78 (2008) 065011 [arXiv:0804.3745] [InSPIRE].

[151] F. Staub, W. Porod and J. Niemeyer, Strong dark matter constraints on GMSB models, JHEP 01 (2010) 058 [arXiv:0907.0530] [InSPIRE].

[152] D. Kazakov, Supersymmetry in particle physics: the renormalization group viewpoint, Phys. Rept. 344 (2001) 309 [hep-ph/0001257] [INSPIRE].

[153] S.K. Vempati, Studies in topics going beyond the standard electroweak model, hep-ph/0203219 [INSPIRE].

[154] L.E. Ibáñez, C. Lopez and C. Muñoz, The Low-Energy Supersymmetric Spectrum According to $N=1$ Supergravity Guts, Nucl. Phys. B 256 (1985) 218 [InSPIRE]. 